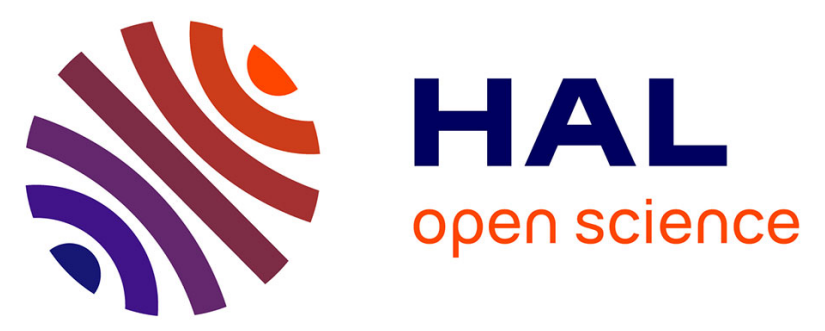

\title{
Évaluation environnementale du PDU nantais 2000-2010 à partir des simulations numériques des scénarios alternatifs du programme Eval-PDU
}

Patrice Mestayer, Bernard Bourges, Laurent Fouillé, Jacques Bougnol, Erwan Bocher, Thierry Schmidt, Judicaël Picaut

\section{To cite this version:}

Patrice Mestayer, Bernard Bourges, Laurent Fouillé, Jacques Bougnol, Erwan Bocher, et al.. Évaluation environnementale du PDU nantais 2000-2010 à partir des simulations numériques des scénarios alternatifs du programme Eval-PDU. RTS - Recherche Transports Sécurité, 2015, Évaluation environnementale des PDU, 2015 (02), pp.97-120. 10.4074/S0761898015002046 . halshs-01192764

\section{HAL Id: halshs-01192764 \\ https://shs.hal.science/halshs-01192764}

Submitted on 14 Mar 2018

HAL is a multi-disciplinary open access archive for the deposit and dissemination of scientific research documents, whether they are published or not. The documents may come from teaching and research institutions in France or abroad, or from public or private research centers.
L'archive ouverte pluridisciplinaire HAL, est destinée au dépôt et à la diffusion de documents scientifiques de niveau recherche, publiés ou non, émanant des établissements d'enseignement et de recherche français ou étrangers, des laboratoires publics ou privés. 


\title{
Évaluation environnementale du PDU nantais 2000-2010 à partir des simulations numériques des scénarios alternatifs du programme Eval-PDU
}

\author{
Environmental assessment of Nantes transportation master plan 2000-2010 \\ from the Eval-PDU program alternative scenario numerical simulations
}

\author{
Patrice Mestayer · Bernard Bourges · Laurent Fouillé · Jacques Bougnol · Erwan Bocher · Thierry Schmidt · Judicaël \\ Picaut
}

Reçu le 16 juillet 2014 ; accepté le 13 novembre 2014

(C) IFSTTAR et Éditions NecPlus 2015

Résumé Le programme de recherche pluridisciplinaire Eval-PDU a proposé une structure d'enchaînement de

\section{Patrice Mestayer $(\square)$ \\ IRSTV FR CNRS 2488, Institut de recherche en sciences et techniques de la ville, LUNAM université, CNRS \\ 1, rue de la Noë, BP 92101, F-44321 Nantes cedex 03, France \\ e-mail : Patrice.Mestayer@ec-nantes.fr}

Bernard Bourges $(\square)$

IRSTV FR CNRS 2488, GÉPEA UMR CNRS 6144, Institut de recherche en sciences et techniques de la ville

École des mines de Nantes, LUNAM université

4, rue Alfred-Kastler, BP 20722, F-44307 Nantes cedex 03, France

e-mail : bernard.bourges@mines-nantes.fr

Laurent Fouillé $(\triangle)$

IRSTV FR CNRS 2488, GÉPEA UMR CNRS 6144, Institut de

recherche en sciences et techniques de la ville

École des mines de Nantes, LUNAM université

4, rue Alfred-Kastler, BP 20722, F-44307 Nantes cedex 03, France

e-mail : 1.fouille@yahoo.fr

Jacques Bougnol $(\square)$

CEREMA, Direction Territoriale Ouest (ex CETE de l'Ouest), MAN rue René Viviani, BP 46223, F-44262 Nantes cedex 02, France

e-mail : jacques.bougnol@cerema.fr

Erwan Bocher $(\bowtie)$

IRSTV FR CNRS 2488, Institut de recherche en sciences et techniques de la ville, LUNAM université, CNRS

1, rue de la Noë, BP 92101, F-44321 Nantes cedex 03, France

e-mail : erwan.bocher@ec-nantes.fr

Thierry Schmidt $(\square)$

Air Pays de la Loire, Association Agréée de Surveillance de la Qualité de l'Air des Pays de la Loire

5 rue Edouard Nignon, 44300 Nantes

e-mail : schmidt@airpl.org

Judicaël Picaut $(\square)$

IFSTTAR, Laboratoire d'Acoustique Environnementale,

Département AME, Route de Bouaye, CS 4, 44344 Bouguenais

Cedex, France

e-mail : judicael.picaut@ifsttar.fr modèles numériques à base physique, calculant le trafic multimodal sur les réseaux, les consommations énergétiques, les émissions de gaz à effet de serre, les concentrations de polluants atmosphériques, leur impact sanitaire, le bruit, et les conséquences sur les prix de l'immobilier. Ces modèles sont reliés par une infrastructure de données spatiales commune qui assure la gestion des données d'un étage de modélisation à l'autre et permet, par croisement de résultats de simulations avec des données de natures différentes, de calculer des indicateurs intégrés synthétiques originaux. Un exercice d'évaluation des impacts environnementaux du Plan de déplacements urbains (PDU) 2000-2010 de Nantes Métropole a été réalisé sur la base de simulations numériques rendant compte d'une part des situations réelles de 2002 et 2008 et d'autre part de scénarios alternatifs représentant des situations virtuelles stratégiques ou des tests de sensibilité aux variables pouvant servir de levier dans la planification des déplacements urbains, telles que les prix, les interdictions, et les aménagements de réseau. Parmi l'ensemble des travaux menés durant le programme de recherche Eval-PDU, cet article présente essentiellement l'évaluation des impacts en termes de pollution atmosphérique et d'émissions de Gaz à effet de serre (GES), sous l'angle de la chaîne de modèles et des résultats obtenus.

Mots clés impacts environnementaux - déplacements . méthodologie · évaluation $\cdot$ politiques urbaines

Abstract The pluridisciplinary research program Eval-PDU
proposed a chaining structure of physically-based, numerical
models computing multimodal traffics on the networks,
energy consumptions, greenhouse gas emissions, atmo-
spheric pollutant concentrations, their health impacts, noise
levels, and their consequences on real estate values. These
models are linked by a common spatial data infrastructure 
that ensures data management from one modelling stage to the others and provides computation algorithms of original synthetic indicators by putting in relation simulation results and data of different natures. The method has been tested to evaluate the environmental impacts of the 2000-2010 transportation master plan (PDU) of Nantes Métropole, based on numerical simulations of the real situations of 2002 and 2008 on the one hand, and of alternative scenarios featuring strategic virtual situations or sensitivity tests of some variables that may be used as leverage in urban transportation planning, such as prices, forbiddances, and network improvements. Among the works brought to a successful conclusion during the research program Eval-PDU, this article presents essentially the impact evaluation as regards atmospheric pollution and GHG emissions, from the point of view of the model chain and the results obtained.

Keywords Environmental impacts · Mobility .

Methodology · Assessment · Urban policies

\section{Introduction}

\subsection{L'évaluation des Plans de déplacements urbains (PDU)}

Nos déplacements en voiture ont des impacts importants sur l'environnement. Ces impacts nous concernent en grande partie localement : c'est le cas de la pollution de l'air par les gaz d'échappement avec ses conséquences sanitaires. D'autres conséquences sont à plus grande échelle, mais également inquiétantes, comme la consommation des énergies fossiles ou les émissions de gaz à effet de serre. Les atteintes à l'environnement causées par les transports sont ainsi devenues une préoccupation du grand public et une thématique essentielle de l'action publique, pour les institutions européennes, l'État ou les villes.

Évaluer les impacts environnementaux des nouveaux équipements et des politiques publiques en matière de transports est donc devenu un impératif.

Des textes réglementaires rendent obligatoire cette évaluation. Il s'agit de prévoir quelles seront les nuisances associées à telle ou telle action, afin de choisir a priori les politiques ayant les incidences les plus faibles. Une nouvelle ligne de Busway, des vélos en libre-service... au-delà du service qu'elles rendent, quelles conséquences environnementales auront ces différentes actions ? Certaines seront-elles préférables ?

Mais l'évaluation peut aussi avoir un rôle a posteriori pour vérifier, une fois l'action réalisée, que les objectifs prévus ont été atteints ou que des conséquences négatives non prévues ne sont pas venues annuler les bénéfices.
La question, alors, est « comment évaluer ces impacts environnementaux, passés ou futurs?»

Les mesures, telles que celles qui sont effectuées par les établissements en charge de la surveillance de la qualité de l'air (Air Pays de la Loire à Nantes) sont indispensables, mais elles ne suffisent pas. Elles ne permettent pas de dire ce qui se passera dans 5 ou 10 ans si on crée une nouvelle ligne de bus. Mais elles ne suffisent pas non plus pour dire si la mauvaise qualité de l'air aujourd'hui est directement la conséquence de tel ou tel choix fait il y a 5 ou 10 ans par la Métropole ; en effet de nombreux facteurs ont changé eux aussi indépendamment des politiques locales, comme la démographie ou le parc automobile. Pour évaluer les conséquences de tel ou tel choix, il n'est pas possible non plus de faire des expériences de laboratoire ! Il n'y a pas d'autres choix que de recourir à des modèles.

Un modèle est une représentation mathématique des phénomènes, implémentée sur ordinateur et qui permet de simuler le fonctionnement du système étudié dans des conditions choisies et bien définies.

Le projet Eval-PDU a été consacré au développement et à l'application d'un ensemble de modèles adaptés à l'évaluation des impacts environnementaux (pollution, gaz à effet de serre, bruit) de différentes actions sur les transports et la mobilité urbaine. Un premier modèle sert à analyser les déplacements dans l'agglomération en fonction de la population et de sa répartition, des lieux d'emploi et d'activité, des voiries et moyens de transport existants. À partir de cette estimation du trafic routier, un deuxième modèle sert à calculer les émissions de polluants par les véhicules. Un troisième modèle permet de suivre la dispersion de ces polluants dans l'atmosphère et leur répartition dans l'Agglomération. Un quatrième modèle s'intéresse au bruit généré par les transports et à sa diffusion dans la ville.

Le projet Eval-PDU a permis de développer des modèles nouveaux, d'en perfectionner d'autres qui existaient déjà et de les adapter à l'évaluation environnementale des actions d'une collectivité. Aucun modèle ne peut rendre parfaitement compte de la réalité, mais si l'on prend garde de les utiliser dans le domaine pour lequel ils sont conçus, ils donnent des informations précieuses et fournissent une bonne idée des tendances.

Évaluer une action dans l'absolu n'a en général pas grand sens : on évalue plutôt de manière comparative les résultats de l'action avec ceux d'une autre action ou absence d'action.

Il faut donc préciser la situation qui est prise comme référence, les actions qu'elle inclut ou non et leurs caractéristiques. L'impact d'une nouvelle ligne de bus dépend du réseau déjà existant, de son fonctionnement, des tarifs, du réseau routier, etc. Mais il dépend aussi de facteurs exogènes comme le prix des carburants ou le parc 
de véhicules. Ceci rend l'évaluation difficile et explique les débats passionnés qu'elle suscite : on peut avoir en tête une seule et même action (le Busway par exemple) mais aussi la comparer à des références très différentes ou l'analyser dans des situations ayant peu de points communs.

L'évaluation ne peut donc procéder que par scénarios et il importe de décrire aussi précisément les scénarios utilisés que les modèles mis en æuvre et leur articulation.

\subsection{Eval-PDU et la modélisation intégrée}

L'origine du programme Eval-PDU réside dans une proposition de Nantes Métropole de mener une recherche méthodologique sur l'évaluation des impacts environnementaux, plurifactoriels, du PDU nantais. Sur la base des compétences que nous pensions pouvoir mobiliser dans les équipes participant à l'Institut de recherche en sciences et techniques de la ville (IRSTV), ou proches, nous avons proposé une étude pluridisciplinaire en vue de la construction d'une méthodologie centrée sur la modélisation de la qualité de l'air et du bruit et associant leurs conséquences socio-économiques. Un programme de recherche-action a été établi en 2007 pour établir les lignes directrices et les concepts fondamentaux de cette démarche pluridisciplinaire, et constituer l'équipe de projet, puis une étude méthodologique générique, dépassant le cadre du PDU de Nantes Métropole mais l'utilisant comme banc-test de son applicabilité a été proposée à l'ANR Villes Durables en 2008 [1,2].

Le concept initial était celui d'une chaîne de modèles à bases physiques permettant de mettre en relation, selon différents scénarios, les déplacements dans l'aire urbaine de la métropole, la qualité de l'air et les nuisances sonores. Dans une perspective plus large d'évaluation des impacts sur le développement durable, notamment en relation avec l'élaboration du Plan climat nantais, l'objectif a été élargi, d'une part aux consommations énergétiques et émissions de Gaz à effet de serre (GES), d'autre part à l'évaluation de certains impacts sur la santé, enfin aux conséquences socio-économiques des impacts environnementaux $[3,4]$.

Avec une volonté de généralisation et d'applicabilité de la méthode proposée, deux tâches complémentaires ont été ajoutées :

1. une analyse de l'adaptation des comportements individuels aux actions du PDU sur la base d'une enquête auprès d'usagers des transports en commun nantais, éclairée par une série d'études sociologiques, politiques et juridiques sur l'impact de «l'environnementalisation » des règles et instruments du droit de l'urbanisme et du droit foncier $[5,6]$;

2. la réalisation d'un retour d'expérience après les tests de scénarios du PDU nantais, permettant de proposer des améliorations et simplifications de la méthode et des préconisations de mise en œuvre [7] ;

Les principaux résultats de la recherche et les préconisations pour une évaluation d'un PDU à forte ambition écologique ont été présentés lors d'un séminaire de restitution [4] et l'ensemble des 24 documents « livrables » (études bibliographiques et rapports méthodologiques) sont librement disponibles (http://www.irstv.fr).

Le schéma envisagé pour la méthode d'évaluation était celui d'une chaîne de modèles à base physique ou statistique, en " cascade », dont chaque étage de modélisation alimente en données d'entrée l'étage suivant (Figure 1). Dans le domaine de la qualité de l'air des zones urbaines la démarche d'intégration de plusieurs modèles dans une suite cohérente a été adoptée depuis de nombreuses années, d'abord pour réaliser des simulations de la dispersion des polluants sur des domaines emboîtés permettant de zoomer sur une petite zone en prenant en compte les contributions de zones plus larges (par exemple Moussiopoulos [8]) à partir de cadastres d'émissions statiques, puis pour intégrer la simulation des émissions et leurs impacts sur la population (par exemple Borrego et al [9]), passant ainsi de l'intégration de modèles homogènes à celle de modèles hétérogènes. Plus récemment, l'intégration d'un modèle de déplacements en amont de la modélisation des émissions par les véhicules a ouvert la voie à la réalisation d'études prospectives par simulation de situations virtuelles $[10,11,12]$. Parallèlement on notera l'intégration des résultats d'un modèle de déplacements à des calculs d'indicateurs d'impacts environnementaux et socio-économiques des transports réalisée par Lautso et Toivanen [13]. On renvoie à Hatzopoulou et al. [12] pour une revue bibliographique internationale et à Broc et Bourges [14], Fouillé et al. [15] et Brutti-Mairesse [16] pour leurs analyses des évaluations environnementales des plans de déplacements réalisées en France et en Europe.

\subsection{L'application de la méthode}

Pour appliquer la méthode Eval-PDU au PDU nantais, plusieurs scénarios ont été élaborés [14, 17]. Tout d'abord deux scénarios historiques ont été choisis s'appuyant sur les situations réelles de l'Agglomération nantaise en 2002 et 2008, tant du point de vue des données socio-économiques, de la voirie et des réseaux de transport publics, que des pratiques de déplacement des habitants. Ces deux scénarios ont permis de caler les modèles et de vérifier que les évolutions constatées entre ces deux dates étaient correctement prises en compte. Par la suite, le scénario de 2008 a servi de référence pour évaluer l'impact de politiques alternatives hypothétiques, toutes choses égales par ailleurs. 
Ces politiques alternatives ont été synthétisées dans trois scénarios :

- Le scénario «PDU volontariste » (ou PDU max) intègre toutes les actions prévues à l'origine dans le PDU nantais, y compris les infrastructures de transports collectifs non encore réalisées en 2008 ou abandonnées [18];

- Le scénario «PDU minimaliste » (ou PDU min), plus favorable à l'automobile et peu tourné vers les transports collectifs, comprend les mêmes infrastructures de transports en commun qu'en 2002 et les infrastructures routières de 2008 ;

- Le scénario « sans Busway », situation 2008 dans laquelle le Busway (Ligne 4) et les modifications de voiries associées n'auraient pas été réalisées.

Par ailleurs, toujours avec la situation 2008 comme référence, plusieurs variantes ont été simulées. Il ne s'agit pas d'évaluer des politiques alternatives à proprement parler mais de tester l'influence de certains paramètres, modifiés de manière isolée, sur les résultats des modèles. Certains de ces paramètres correspondent à des actions politiques particulières, qui auraient été appliquées isolément, concernant la voiture (prix du stationnement), ou les transports en commun (prix ou fréquences). D'autres variantes traduisent plutôt la sensibilité à des facteurs exogènes en partie hors de portée de l'action de la collectivité, tels que l'augmentation de la demande de déplacements ou le doublement du prix des carburants.

Quelques résultats marquants de la simulation de ces scénarios par nos modèles sont présentés et discutés ci-dessous. À la lecture de cet article, on gardera à l'esprit les remarques qui précèdent : il s'agit d'éléments d'information pour alimenter le débat sur les politiques de transport et de mobilité en ville, certainement pas des chiffres absolus. Au-delà d'indications globales à l'échelle de l'Agglomération, les informations plus locales (quartier ou voie) sont souvent les plus intéressantes.

\section{Le programme Eval-PDU}

\subsection{La construction de la méthode}

Au cœur du dispositif initialement esquissé se situent les modèles de calcul des concentrations de polluants réglementés dispersés dans l'atmosphère d'une part, des cartes d'indices de nuisance sonore au sol et au niveau des bâtiments d'autre part. Les principales données d'entrée de ces modèles sont des cadastres d'émissions, cartes spatio-temporelles des sources de polluants et de bruit. Pour obtenir ces cadastres, une modélisation des flux de trafic est nécessaire en amont. Elle est produite à l'aide d'un modèle multimodal des déplacements, dont les

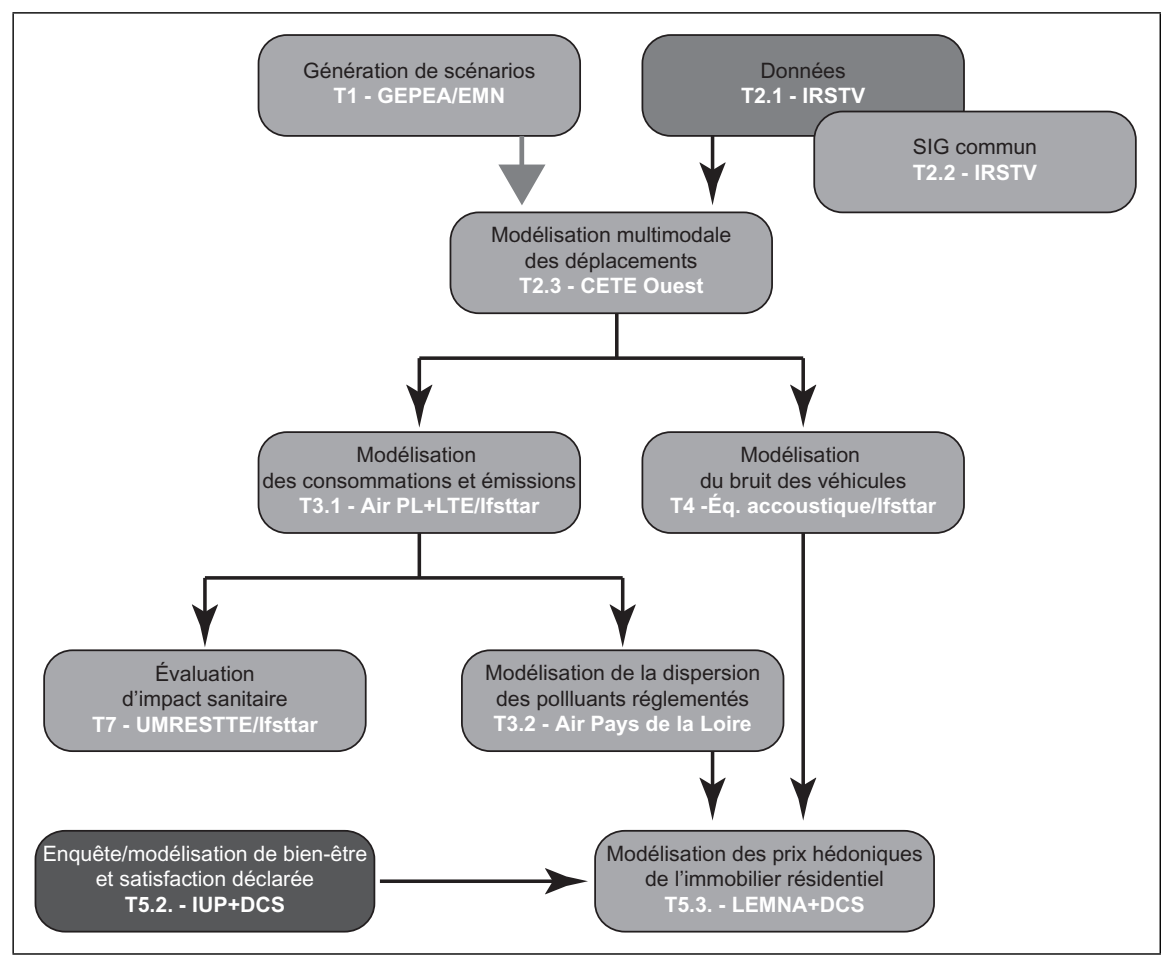

Fig. 1 Schéma de la méthode de modélisation « en cascade », identifiant les différentes tâches du programme, les équipes participantes (voir les abréviations dans les remerciements) et les flux de données (flèches) 
principales données d'entrée sont d'une part l'offre de transport, d'autre part les données socio-économiques du territoire concerné, des enquêtes ménages déplacements et des comptages routiers. L'élargissement de l'objectif à l'évaluation explicite des consommations énergétiques et des émissions de GES nous a amenés à séparer l'étape de modélisation des consommations et des émissions (de GES et de polluants) entre celle des déplacements et celle de la dispersion des polluants dans l'atmosphère, d'autant qu'il était décidé de construire une méthode alternative d'évaluation de l'impact sanitaire des émissions polluantes des transports, alimentée par la modélisation des émissions et basée sur la construction d'un indicateur spécifique.

En aval des calculs de concentration de polluants et de niveaux de bruit, la contribution au bien-être des populations est estimée en parallèle par deux analyses géostatistiques multivariées, l'une basée sur les déterminants des prix de l'immobilier au travers d'un modèle de prix hédoniques appliqué aux transactions immobilières, l'autre sur les résultats d'une enquête sur le bien-être et la satisfaction déclarée des habitants, interrogés à leur domicile.

Plus que celui d'une chaîne linéaire, ce dispositif apparaît comme un schéma de « cascade » dont chaque étage de modélisation alimente en données d'entrée les étages suivants (Figure 1). Dans ce schéma le dispositif est alimenté en données réelles pour les années de référence pour lesquelles elles existent et pour lesquelles des validations sont donc possibles, et en données virtuelles permettant de simuler les variations d'impacts environnementaux générées par des scénarios alternatifs. Enfin un Système d'informations géographiques (SIG) commun permet de gérer les données et éditer les différents résultats de manières cohérentes.

Le schéma de la Figure 1 semble supposer que les transferts de données transitant d'une étape de modélisation aux suivantes sont simples, monodirectionnels et sans ambiguiités. Nous savions qu'il n'en était rien et que le succès d'un tel dispositif repose largement sur l'acquisition, la transmission et la cohérence des données. C'est pourquoi une tâche spécifique avait été prévue pour l'acquisition et le management des données dans une infrastructure de données spatiales commune appuyée sur le SIG communautaire.

\subsection{La gestion des données}

Pour faciliter les échanges de données géographiques et leur manipulation, mais aussi pour garantir leur cohérence et leur unicité, nous avons construit une Infrastructure de données spatiales (IDS). Cette IDS nommée CartoPolis est un système d'information composé de 5 modules interdépendants (Figure 2) [20].

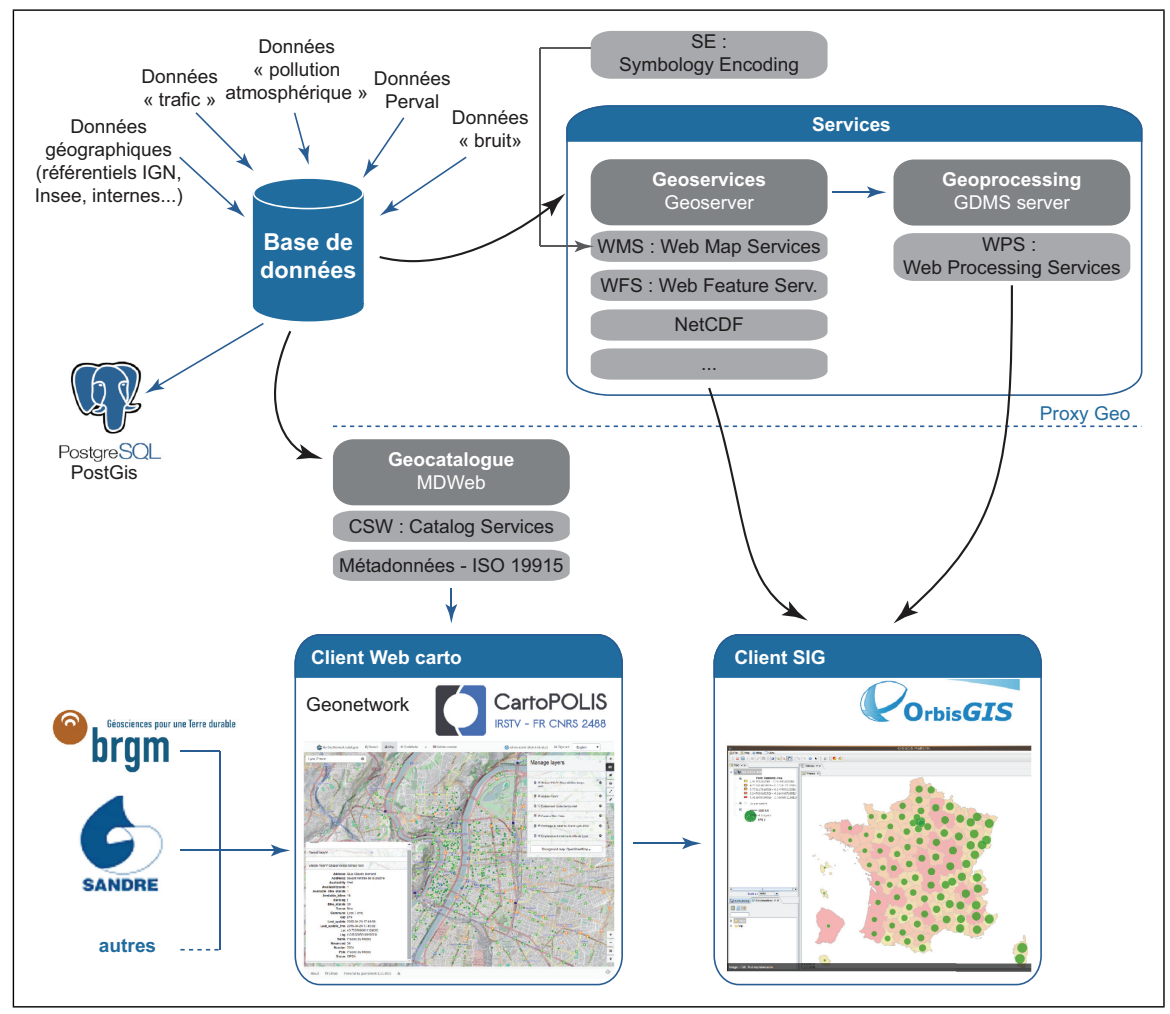

Fig. 2 Architecture et composants de l'infrastructure de données spatiales CartoPolis 
- La base de données unique agrège l'ensemble des données collectées (référentiels géographiques (IGN) et de population (Insee), réseaux routiers et ferrés, enquêtes ménages-déplacements, comptages routiers, fréquences des Transports en commun (TC), base de données Perval des notaires, etc.) ainsi que les données produites par chaque étage de modélisation. Les données sont stockées de manière structurée à l'aide d'un système de gestion de bases de données relationnelles.

- Le Géocatalogue est un ensemble de fiches de métadonnées, à raison d'une fiche pour chacun des jeux de données intégrés dans la base de données unique. Il s'agit de données sur la donnée, structurées selon la norme ISO 19115 (www.isotc211.org) et contenant des informations telles que l'étendue temporelle du jeu de données, son emprise spatiale, son origine, sa qualité sémantique et spatiale, etc. Le Géocatalogue permet également de gérer la visibilité de la fiche (public, restreint à un groupe, interne...), de gérer les droits sur les données, de définir des contraintes d'intégrité, de moissonner d'autres catalogues.

- Le Géoservice est un serveur de flux de données, application permettant de mettre à la disposition des utilisateurs les données géographiques de la base de données, sous la forme d'images cartographiques ou de géométries, sur différents supports (PC, tablette, smartphone) via l'Internet.

- Le portail internet cartographique disposant d'interfaces graphiques permet de questionner le Géocatalogue : l'utilisateur peut y saisir un mot pour rechercher une donnée ou encore rechercher des données selon une étendue spatiale ou temporelle.
- Le SIG communautaire, OrbisGIS, pièce maîtresse du système CartoPOLIS, permet de récupérer les données dans la base de données unique et de réaliser des requêtes de transformation, de calcul et d'édition de ces données $[20,21]$.

Une attention particulière a été apportée à ce dernier module. OrbisGIS, logiciel libre développé en interne par l'IRSTV pour la recherche urbaine depuis une dizaine d'années, a subi d'importants développements méthodologiques et techniques spécifiques permettant, notamment, le couplage de certains logiciels du projet :

- l'intégration d'une approche simplifiée pour la production de cartes de bruit, qui a nécessité la réécriture de l'architecture de sa librairie logicielle et l'optimisation de ses algorithmes pour autoriser par exemple l'écriture de fichiers de données de plusieurs gigaoctets, l'intégration d'une librairie de triangulation Delaunay, etc. ;

- l'amélioration de la production des cadastres de qualité de l'air (méthode de triangulation avec « rasterisation ») ;

- la création de nouvelles requêtes d'analyse spatiale pour les variables géolocalisées des calculs économétriques et de l'impact du report modal du Busway;

- la définition de modes de représentation des cartographies thématiques produites, en vue de leur uniformisation, grâce à l'utilisation de la norme Symbology Encoding.

\subsection{La « méthode Eval-PDU »}

Pour constituer l'ensemble de la méthode les modèles suivants ont été retenus par les équipes participantes (Figure 3).

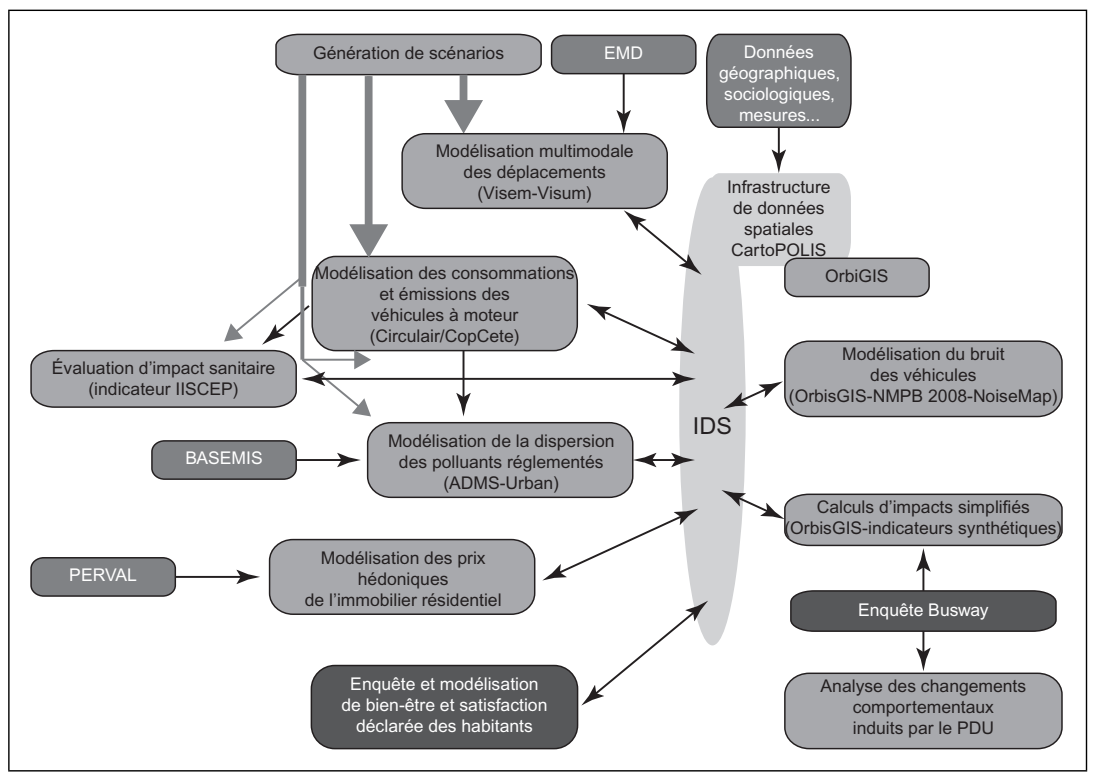

Fig. 3 Schéma de l'articulation des modèles et calculs mis en place dans la « méthode Eval-PDU » 
- Le modèle Visem-Visum distribue, selon la demande évaluée sur la base d'une Enquête ménages déplacements (EMD), les flux de trafics sur les axes des réseaux routier et ferré : véhicules individuels, poids lourds, transports en commun (bus, tramways, Busway, TER). C'est un modèle de déplacements multimodal classique, statique, macroscopique, à 4 étapes : génération de l'offre (zones et réseaux), distribution selon une matrice originedestination (O-D) de zone à zone, choix modal, affectation attribuant un itinéraire à chaque déplacement [15].

- Pour le calcul des consommations énergétiques et des émissions de polluants des véhicules on a retenu le logiciel Circul'Air [24] développé et mis à disposition par l'Association pour la surveillance et l'etude de la pollution atmosphérique en Alsace (ASPA), Association agréée de surveillance de la qualité de l'air (AASQA) de la Région Alsace. Basé sur la méthodologie Computer Program to calculate Emissions from Road Transport (COPERT) 4 [23], ce logiciel intègre un module de calcul de la vitesse horaire moyenne de circulation nécessaire à l'utilisation des équations COPERT. Il propose une palette de résultats : émissions pour chaque polluant et chaque sous-catégorie de véhicules, estimées sur un tronçon, sommées pour un type de tronçon, ou encore totalisées sur une zone d'intérêt. On peut également différencier les émissions selon leur origine : émissions à chaud, surémissions à froid, usure et remise en suspension des particules, évaporation de carburant, etc. [24]

- La sélection du modèle de dispersion atmosphérique a reposé sur une analyse de huit études d'inter-comparaison de modèles urbains ou routiers. Cette analyse, conduite en 2009 dans le cadre de l'élaboration d'un guide de modélisation en milieu urbain [25], a été menée par un groupe d'experts au sein des AASQA animé par Air Pays de la Loire (AirPL). Le modèle retenu pour Eval-PDU est Atmospheric Dispersion Modelling System (ADMS) Urban [26, 27] qui permet de calculer et cartographier les concentrations des polluants atmosphériques réglementés en tenant compte de la météorologie journalière et des émissions de sources ponctuelles, linéiques ou surfaciques, renseignées par Circul'Air pour le secteur routier et par un inventaire d'émissions des secteurs non-routiers (résidentiel, tertiaire, industrie) tel que BASEMIS construit par AirPL à l'échelle de la Région des Pays de la Loire [28].

- Dans l'objectif de réaliser des simulations comparatives de scénarios alternatifs plutôt que la construction de cartes de bruit très exactes en valeurs absolues mais très coûteuses en temps de calcul, on a construit une méthode «simplifiée » pour la modélisation des sources sonores, les calculs de propagation du bruit dans l'environnement bâti et la cartographie des indices de nuisance sonore. Cette méthode s'appuie sur une approche bidimensionnelle (calculs dans le plan horizontal), inspirée de la méthodologie réglementaire française Nouvelle méthode de prévision de bruit (NMPB) 2008 [29], sur une simplification des sources sonores, les tronçons routiers et tramway étant assimilés à des sources composites ponctuelles, et sur une discrétisation de l'espace au sol et devant les façades des bâtiments (points récepteurs). Pour les calculs de propagation acoustique (directe, réfraction, réflexions) un réseau de points sources est construit pour chaque point de calcul en tenant compte de la présence des bâtiments. Les calculs de cette méthode simplifiée sont intégrés dans le système d'information géographique OrbisGIS sous la forme d'un module NoiseMap, ce qui permet une optimisation des algorithmes de production des cartes de bruit [30, 31].

- Pour évaluer les impacts sanitaires chroniques des émissions de polluants un nouvel indicateur a été créé en s'appuyant sur les méthodes identifiées par l'action COST 356 «EST - Towards the definition of a measurable environmentally sustainable transport » [32]. L'indicateur Chronic health impact indicator of pollutant emission (IISCEP) est obtenu en pondérant les émissions de chaque polluant automobile et de chaque zone source par un facteur de toxicité (carcinogène ou non-carcinogène) propre à chaque substance et par un facteur représentant globalement la dispersion atmosphérique et la population exposée de la zone [33].

- Les conséquences des impacts environnementaux sur le bien-être des populations sont estimées au travers d'un modèle de prix hédoniques de l'immobilier prenant en compte les caractéristiques intrinsèques des logements, des variables de localisation et d'accessibilité, notamment la proximité des axes routiers et des transports en commun comme celle des espaces verts, des variables contextuelles et des variables de qualité environnementale, bruit et pollution atmosphérique. Le modèle est appliqué à partir de la base de données Perval des transactions immobilières enregistrées par des notaires [34].

Le schéma de la Figure 3 met en relief une nouvelle articulation entre les modèles et l'infrastructure de données spatiales : même si subsistent quelques transferts directs et privilégiés entre certains étages de modélisation, les flux de données sont assurés de manière externe par l'IDS qui permet à chaque compartiment d'obtenir en entrée les données dont il a exactement besoin en cohérence avec les autres compartiments (qu'ils se situent «en amont» ou « en aval »). CartoPOLIS ne se contente pas d'être l'unique entrepôt des bases de données mais c'est également un instrument de transmission et de gestion de ces données, à l'aide du SIG OrbisGIS, qui permet d'en assurer l'unicité et la cohérence. L'IDS permet aussi de gérer des flux de données multi-directionnels, et non plus 
seulement dans le sens amont-aval du schéma initial, ce qui permet la mobilisation de fonctionnalités des modèles qui n'avaient pas été prévues au départ. Prenons l'exemple des calculs de facteurs d'exposition de la population. Le croisement des données de répartition géographique des habitants (nombre de résidents par Îlot regroupé pour des indicateurs statistiques (IRIS) de l'Insee) avec les résultats des calculs de bruit et d'émissions ou de concentrations permet, à l'aide des outils géomatiques de Cartopolis, de quantifier et de géolocaliser les populations soumises aux impacts environnementaux, par exemple dans les calculs d'IISCEP [33] comme dans ceux des populations soumises à des dépassements de seuils (Section 3.4). Mais il est évident que les habitants ne sont pas dans leur résidence à tout moment de la journée, du fait justement de leurs déplacements, ce qui introduit indéniablement un biais sur nos résultats actuels. En réalité le modèle multimodal de déplacements, qui calcule les transferts entre zones, permettrait non seulement de construire les cadastres de flux (trafic) mais aussi d'évaluer des distributions statistiques des populations dans chaque zone à chacune des périodes de la journée simulée : un « retour dynamique » vers ce modèle amont permettrait donc de largement réduire ce biais en prenant en compte la population réellement impactée dans chaque tranche horaire (cela supposerait toutefois de réaliser des modélisations horaires des déplacements sur une journée type, voire sur une semaine type ou alors de les extrapoler à partir du travail qui a été produit sur quatre tranches horaires représentatives d'un jour).

Ce schéma met également en relief les modélisations qui ont été intégrées à OrbisGIS : le bruit sous la forme du module NoiseMap [30] et les indicateurs synthétiques sous la forme de requêtes accessibles à tous les utilisateurs : calculs de parcours viaires, de distances et temps sur parcours, opérateurs d'intégration spatiale, de comparaison, etc. [35] (Section 2.4). Cette démarche se révèle extrêmement efficace lorsqu'il s'agit de prolonger les études, notamment en testant de nouveaux scénarios. Sans être généralisable elle pourrait être étendue plus largement, par exemple aux calculs économétriques ou d'impact sanitaire, tandis que la souplesse de construction de requêtes externes dans CartoPOLIS permet d'envisager des couplages avec les autres modèles.

Sur ce schéma on note enfin que les notions de compartiments « amont » et «aval» perdent une partie de leur sens puisque des scénarios peuvent s'appliquer à plusieurs de ces compartiments indépendamment de ceux qui figurent plus en amont dans le schéma de la Figure 1 (par exemple des scénarios de changements technologiques ou de réduction de facteurs d'émissions, ou des scénarios «non-PDU » stricto sensu tels que des dispositifs anti-bruit ou des changements climatiques), ou lorsqu'on met en œuvre une méthode alternative ne faisant pas appel directement aux modélisations mais combinant des données-résultats disponibles dans l'IDS à des données autogènes acquises de manière ciblée, comme dans l'exploitation de l'enquête consacrée aux usagers du Busway.

C'est tout particulièrement vrai dans la perspective, évoquée dans la Section 4, de l'évaluation en accompagnement in itinere (chemin faisant) des politiques de mobilité. Si l'on conçoit que ce dispositif n'est pas destiné à servir une seule fois, dans un temps relativement court juste avant le vote du Conseil communautaire, mais à accompagner le déroulement du plan et à en évaluer les phases en cours de route, voire à préparer dès son démarrage sa future évaluation finale, alors la «méthode Eval-PDU » se lit différemment. Les différents étages de modélisation n'apparaissent plus comme des étapes successives de travail, les relations de dépendance temporelle disparaissent et les seules relations de dépendance qui subsistent sont celles de la présence dans l'IDS des données nécessaires aux étages de modélisation effectivement mis en œuvre pour réaliser le scénario. Une phase initiale de production des situations de référence reste indispensable, mais l'IDS est alors prête à alimenter de nombreuses variantes des scénarios, à un coût modéré.

\subsection{Une « méthode simplifiée » d'indicateurs synthétiques}

La « méthode Eval-PDU » proposée, qui réclame l'intervention de spécialistes des différents types de modélisation, s'éloigne des méthodes plus légères adoptées précédemment par les évaluateurs des PDU. C'est pourquoi une « méthode simplifiée » de calcul d'indicateurs d'impact « globaux » a été également proposée pour comparer les impacts des différents scénarios en minimisant les calculs. Elle est basée sur les résultats des simulations du trafic auxquels sont appliqués des calculs d'intégration spatiale et temporelle d'une part et des calculs très simplifiés de consommation d'énergie, émission de GES et émissions de polluants réglementés [35].

Le calcul est établi pour l'ensemble d'un territoire (par exemple celui de Nantes Métropole) et pour une journée type de semaine. L'intégration de tous les déplacements sur 24 heures permet de calculer la distance totale parcourue par les véhicules à moteur, le temps total de déplacement et la vitesse moyenne. Par comparaison à la simulation des vitesses sur le réseau « à vide » on obtient, par différence des temps totaux de déplacement, un quatrième indicateur appelé « Heures perdues ». On peut également en déduire un pourcentage de ralentissement. Ces deux derniers calculs reposent sur une hypothèse d'invariance des itinéraires sur le réseau « à vide » et « en charge »: les pertes de temps 
sont estimées en comparant les vitesses pratiquées en charge (du fait de la congestion) avec ce qu'elles seraient avec un réseau à vide (vitesse limite nominale). Toutefois, avec cette hypothèse simplificatrice on considère que les itinéraires pratiqués n'ont pas changé avec la congestion ; en réalité les pertes de temps sont encore plus importantes pour tous ceux qui ont quitté l'itinéraire le plus rapide sur le réseau à vide.

Les calculs de consommation d'énergie (carburant) et d'émissions totales s'appuient alors sur les simplifications suivantes : on considère uniquement les émissions 1 . liées à la combustion, 2. à chaud, 3. avec un facteur de consommation moyen pour tout le parc circulant et 4 . un facteur d'émission unique pour chaque polluant. On obtient alors 10 indicateurs, ou 11 avec le pourcentage de ralentissement mais ce dernier contient la même information que les « Heures perdues ». Ces hypothèses de simplification sont toutes basées sur l'observation des résultats des simulations détaillées.

Les valeurs de ces indicateurs intégrés sont exprimées en unités très différentes ; par exemple pour la situation de référence 2008 de Nantes Métropole, la circulation journalière représente $12196613 \mathrm{~km}$ parcourus, 231183 heures de déplacements, $52,76 \mathrm{~km} / \mathrm{h}$ de vitesse moyenne, 29407 heures perdues, 12,5 tonnes de NOx, 10,1 de $\mathrm{CO}, 1,9$ de COV, 0,566 de particules, 780,9 tonnes de carburant et 2 480,9 tonnes de CO2 final. Pour obtenir des indices comparables normalisés en \% on divise les valeurs des indicateurs de chaque scénario par celles de la situation de référence.

Cette méthode de calcul est beaucoup plus rapide que la méthode complète de la chaîne de modélisations, mais elle repose sur des hypothèses simplificatrices et elle ne permet de mettre en lumière ni les effets non-linéaires des mesures mises en œuvre, ni leurs impacts localisés. Par ailleurs, du fait d'échelles temporelles différentes, (un jour type décomposé en 4 périodes pour la méthode simplifiée et une distribution horaire sur les 365 jours de l'année avec Circul'Air), il est possible de voir d'apparentes incohérences entre les résultats obtenus avec ces deux méthodes.

\section{Mise en ouvre et tests sur le PDU nantais, résultats marquants}

\subsection{Application au PDU nantais}

Nantes Métropole est une communauté urbaine regroupant 24 communes de Loire-Atlantique, en région Pays de la Loire. Située à l'ouest de la France, elle s'étend sur plus de $523 \mathrm{~km}^{2}$. Elle est traversée par un fleuve, la Loire, et deux rivières, l'Erdre et la Sèvre. Elle compte 590000 habitants, la moitié résidant à Nantes. Selon l'Agence d'urbanisme de la région nantaise (AURAN) [36] le territoire voit plus de 2 millions de déplacements quotidiens, $57 \%$ s'effectuant en voiture et $15 \%$ en transports collectifs, pour une distance parcourue moyenne de $5 \mathrm{~km}$ et un temps de trajet moyen domicile- travail de 25 minutes. Nantes a été désignée « capitale verte de l'Europe » en 2013 grâce aux bons résultats environnementaux affichés sur des critères relatifs aux transports, à la qualité de l'air, à l'exposition sonore, à la gestion des déchets et à l'aménagement d'espaces verts dans la ville.

Le périmètre d'étude est celui des 24 communes de Nantes Métropole. Pour tenir compte du trafic de transit les calculs de déplacements portent sur un domaine nettement plus étendu, correspondant approximativement au département de Loire-Atlantique (1 936000 déplacements) découpé, pour calculer les matrices origines-destinations, en 397 zones (229 IRIS pour les 24 communes de Nantes Métropole et des regroupements d'IRIS ou de communes au-delà) (Figure 4a). Les réseaux sont modélisés en 5400 tronçons, dont 4100 sur l'aire urbaine de Nantes (Figure 4b). Les déplacements sur le réseau sont calculés explicitement et les déplacements intra-zones sont estimés. Les modes piéton, véhicule individuel, Transport express régional (TER) et Transport collectif (TC) urbain sont calculés (pas les deux-roues) et les poids lourds sont pris en compte. Les calculs de trafic (nombre de véhicules de chaque type et vitesse moyenne horaire sur chaque tronçon) sont effectués sur 4 périodes horaires d'un jour ouvrable : période de pointe matinale ( $7 \mathrm{~h}-9 \mathrm{~h})$, période de pointe vespérale (17h-19h), période creuse nocturne (20h-6h) et période creuse diurne représentant tout le reste de la journée (6h-7h, 9h-17h, 19h-20h).

En sortie du modèle de déplacements les informations en moyennes horaires sur les 4 périodes horaires imposent d'utiliser pour les calculs de bruit des indicateurs de type niveau de pression acoustique continu équivalent (Leq,T) ou de type composite sur les 4 périodes proposées. La reconstitution, grâce à l'utilisation d'un profil type de trafic routier, des données de trafic sur les intervalles de référence de la législation française et européenne en matière de bruit, à savoir $6 \mathrm{~h}-18 \mathrm{~h}, 18 \mathrm{~h}-22 \mathrm{~h}$, et $22 \mathrm{~h}-6 \mathrm{~h}$, permet de calculer des indicateurs standards, de type Lden, Lnight, Levening et Lday [30]. Pour chaque scénario les calculs de propagation sont effectués d'une part pour chaque parcelle de la base de données Perval de l'agglomération nantaise et son environnement immédiat pour alimenter le modèle de prix hédoniques, d'autre part pour l'ensemble de l'espace et des immeubles pour établir des cartes de bruit comparatives. Une méthode d'affectation des nombres d'habitants à chaque immeuble à partir des données Insee à l'IRIS et des données de la BDTopo de l'IGN, construite dans OrbisGIS, permet également de les croiser avec les calculs de bruit pour établir des cartes des populations impactées. 


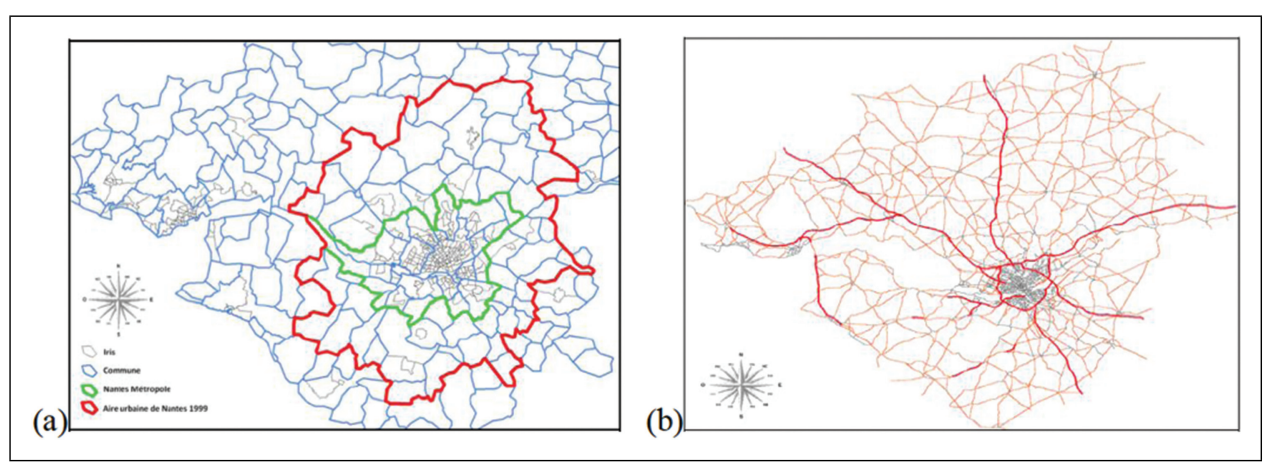

Fig. 4 Domaine de simulation des déplacements : (a) découpage des zones représentant les points d'origine et de destination des trajets ; (b) réseau routier structurant (5 500 arcs)

Les calculs de consommations et émissions sont réalisés sur chaque tronçon du réseau routier, d'une part sur les 4 périodes horaires du modèle de déplacements, d'autre part à l'échelle annuelle en extrapolant ces 4 périodes horaires d'un jour ouvrable hors vacances aux différents types de jours (week-end, vacances scolaires) à partir de l'exploitation des comptages routiers permanents. Les polluants pris en compte sont les suivants : $\mathrm{CO}, \mathrm{NOx}, \mathrm{COVNM}$ (dont benzène, toluène, xylène), formaldéhyde, $\mathrm{SO} 2, \mathrm{CH} 4$, $\mathrm{CO} 2, \mathrm{~N} 2 \mathrm{O}, \mathrm{NH} 3, \mathrm{PM} 10, \mathrm{PM} 2,5, \mathrm{PM} 1$, particules totales, HAPs, POP, dioxines, furanes, et métaux toxiques. Les facteurs de consommation de carburant et d'émission des différents polluants proviennent du guidebook EMEP CORINAIR. Les calculs de concentrations de polluants prennent également en compte les émissions du secteur résidentiel/tertiaire (chauffage) et de 29 sites industriels, figurant dans BASEMIS. Les données météorologiques proviennent de la station de Météo France de l'aéroport Nantes Atlantique. Les concentrations après dispersion sont calculées sur une grille régulière à maille de $250 \mathrm{~m}$ $(\approx 10000$ points $)$ et pour environ 50000 points à proximité immédiate des axes routiers, soit 112 points au $\mathrm{km}^{2}$ en moyenne et 194 points au $\mathrm{km}^{2}$ dans la zone intra-périphérique. L'utilisation de la méthode d'affectation des habitants développée dans OrbisGIS permet également d'établir des cartes des populations impactées par les niveaux de pollution.

Les résultats des calculs de l'indicateur d'impact des émissions sur la santé et du modèle de prix hédoniques des propriétés ayant été présentés ailleurs [33,37,38], ils ne sont pas détaillés ici. En résumé les comparaisons des IISCEP de 2002 et 2008 montrent les points suivants :

- L'impact sanitaire chronique est très majoritairement (de 82 à $90 \%$ ) dû aux particules fines, dans une moindre mesure (de 16 à $9 \%$ ) aux Composés organiques volatiles non méthaniques (COVNM), de 1 à $2 \%$ au formaldéhyde et très nettement moins $(<0,1 \%)$ aux autres polluants.
- À l'échelle de la Métropole, l'IISCEP a diminué de $28 \%$ alors que le nombre de $\mathrm{km}$ parcourus a augmenté de $14 \%$, diminution due en partie au PDU mais surtout à l'évolution du parc roulant.

- Cette décroissance n'est pas également répartie sur l'agglomération : certaines zones périurbaines moins habitées connaissent une légère dégradation $(7 \%$ des zones cumulent une augmentation de $0,8 \%$ de l'impact), tandis que quelques zones centrales où l'impact sanitaire à long terme est potentiellement le plus élevé (fortes émissions, population importante) bénéficient d'une forte amélioration : $12 \%$ des zones cumulent $50 \%$ de la diminution de l'impact. [33]

En ce qui concerne les conséquences sur les prix du marché immobilier, le modèle montre que l'accessibilité au centre grâce aux transports collectifs urbains joue un rôle non négligeable mais contrasté selon la localisation près du centre ville ou sur la périphérie. L'étude a mis en relief l'importance de certaines variables environnementales telles que la proximité des espaces verts. L'exposition au bruit diminue significativement mais faiblement le prix au $\mathrm{m}^{2}$ : par rapport à l'appartement de référence exposé à un bruit de $62 \mathrm{dBA}$ on observe une dépréciation de seulement $0,3 \%$ par décibel supplémentaire. Le niveau moyen des polluants dans l'air n'explique pas de façon significative le prix au $\mathrm{m}^{2}$; par contre on observe des effets de seuil : les logements bénéficiant d'une concentration maximale en NOx inférieure au seuil de $90 \mu \mathrm{g} / \mathrm{m}^{3}$ voient leur prix augmenter significativement, de $6,7 \%$ par rapport à l'appartement de référence. [37, 38]

Les résultats de l'enquête sur le bien-être et la satisfaction déclarée des habitants, interrogés au domicile, n'ayant pas été exploités, une autre enquête a été réalisée dans le Busway (Ligne 4) en vue d'estimer les effets du report modal (voiture vers Busway) à partir d'informations collectées auprès de la population des passagers en situation avérée de choix modal [39]. 


\subsection{Construction des scénarios}

La conception des scénarios pour l'évaluation des impacts environnementaux de PDU s'appuie sur trois grandes catégories d'éléments [14].

- D'une part, les concepts de l'évaluation conduisent à distinguer premièrement l'évaluation des actions d'un PDU au sens de l'analyse de leur logique d'intervention (mise en œuvre), deuxièmement l'évaluation des effets des actions du PDU sur les déplacements, et troisièmement l'évaluation des impacts environnementaux de ces effets.

- Pour l'application de modèles de simulation à ces deux derniers niveaux d'évaluation, deux objectifs peuvent être identifiés. D'abord, l'évaluation stricto sensu des impacts des actions, destinée notamment aux acteurs du processus PDU, doit prendre en compte de véritables scénarios cohérents d'actions sur la mobilité. Mais une étape intermédiaire est nécessaire pour tester l'influence des différents paramètres des modèles et leur fiabilité, mieux comprendre les chaînes de causalité, donner une meilleure vision aux parties prenantes (évaluateurs et acteurs) des atouts et des limites des modèles qu'ils utilisent. Des analyses de sensibilité, portant sur des paramètres isolés, constituent l'outil privilégié de cet objectif intermédiaire.

- Enfin, sur le plan de la définition des scénarios pour la simulation, il convient de distinguer deux niveaux. Premièrement, la définition des scénarios à un niveau général (champs et périmètre d'étude, référentiel, groupes d'action) : elle doit se faire plutôt dans la logique des acteurs, à qui sont destinés in fine les résultats, mais en respectant les contraintes des modèles. Deuxièmement la spécification des jeux précis de données pour la modélisation (paramètres, sources, échelles, hypothèses) : c'est principalement l'affaire des modélisateurs en concertation avec les acteurs.

Le travail préalable s'appuie sur une analyse des possibilités des modèles de déplacements pour traduire les effets d'actions de PDU, ainsi que sur la définition de typologies des actions, issue de l'analyse du bilan des actions du PDU nantais 2000-2010. Les scénarios généraux sont centrés sur les hypothèses fortes (choix des actions étudiées, évolutions structurantes des réseaux, etc.), fondés sur des analyses amont (analyse de la logique d'intervention, etc.) et définis avec une concertation étroite entre le comité de pilotage (représentant les acteurs) et l'équipe d'évaluation (modélisateurs). En outre, ces scénarios généraux sont complétés par des analyses de sensibilité qui sont elles-mêmes reliées à des actions élémentaires sur la mobilité.
Une synthèse des matériaux disponibles concernant l'évaluation du PDU 2000-2010 de Nantes Métropole et de ses actions a été établie sur la base des documents provenant de Nantes Métropole, l'AURAN, deux bureaux d'étude, le Conseil de développement de Nantes Métropole. Elle présente un bilan et une typologie des actions du PDU, les déterminants et grandes tendances des déplacements, les évolutions par mode de déplacement, les efforts d'évaluation réalisés et les limites des résultats présentés [17]. Cette synthèse permet de faire ressortir les éléments structurants pour la définition de scénarios. Les grandes tendances à prendre en compte, les actions phares à intégrer et les questions en débat sont ainsi identifiées. En parallèle, les contraintes et les opportunités de la chaîne de modèles pour l'évaluation des impacts environnementaux sont analysées, en premier lieu celles liées au modèle de trafic. Nous pouvons ainsi mettre en lumière la faisabilité (ou non) de la modélisation des principales actions du PDU ou des grandes tendances les plus significatives. En prenant en compte le nombre de simulations réalisables par les équipes sur la durée du programme, le croisement de ces deux dimensions d'analyse débouche sur une proposition de quelques scénarios d'évaluation, en distinguant des scénarios stratégiques complets et des analyses de sensibilité limitées à une variable. Les indicateurs synthétiques de résultats associés sont également traités.

\subsection{Définition des scénarios étudiés}

La définition et la sélection des situations calculées ou simulées ont été co-construites avec les modélisateurs, puis débattues avec tous les chercheurs et les acteurs opérationnels de Nantes Métropole au cours d'un séminaire commun, avant d'être avalisées. Deux situations réelles ont été retenues, 2002 et 2008, et trois scénarios stratégiques impliquant toutes les étapes de modélisation des déplacements, tandis que huit scénarios-tests de sensibilité ne portent que sur certaines d'entre elles, sans remettre en cause l'offre établie pour la Référence 2008. Tous les scénarios alternatifs sont basés sur la Référence 2008 pour faire apparaître des différences par rapport à la situation réelle de 2008.

Bien que le PDU ne soit terminé qu'en 2010 et que l'intervalle 2002-2008 soit relativement court, l'année 2008 est choisie comme référence car c'est la dernière année du PDU pour laquelle on dispose de données fiables de déplacements. En l'absence de nouvelle enquête ménages déplacements, le paramétrage initial du choix de mode, calé sur l'enquête de 2002, est conservé. De 2002 à 2008 on note l'augmentation de $6 \%$ de la population (579 000 habitants en 2006), de 8,4\% des déplacements (2061000), et de $22 \%$ de l'offre kilométrique de transports en commun. Sur le réseau de Nantes Métropole, l'accroissement estimé 
des véhicules-km est de l'ordre de $17 \%$ aux Heures de pointe du matin (HPM), de $10 \%$ seulement aux Heures de pointe du soir (HPS) et de $14 \%$ sur la journée. La part modale (\% des déplacements des habitants de Nantes Métropole) de la voiture individuelle augmente de 61,6 à $62,6 \%$ pour les déplacements à l'intérieur de Nantes, et de 86,6 à $88,2 \%$ hors périphérique. Les transports en commun urbains (196 000 déplacements sur le réseau urbain) sont en recul d'un point en part modale (10,5 à 9,5\%) malgré une augmentation en nombre de déplacements. Le nombre de sections de voies saturées à plus de $90 \%$ passe de 50 à 76 en HPM et de 72 à 75 en HPS. La vitesse moyenne chute de 51 à $47 \mathrm{~km} / \mathrm{h}$ en HPM et de 49 à $45 \mathrm{~km} / \mathrm{h}$ en HPS.

Après les trois lignes de tram, puis leurs prolongements, la Ligne 4 Busway est une opération particulièrement emblématique du PDU nantais. Il s'agit du remplacement d'une pénétrante majeure en boulevard urbain accueillant l'un des premiers Bus à haut niveau de service (BHNS) français en site propre. Cet axe était la principale pénétrante sud de l'agglomération, dans l'axe de l'autoroute A83 et des nationales vendéennes, franchissant la « seconde ligne de ponts », et aboutissant en plein centre au pied du château des Ducs de Bretagne. Elle est remplacée par un boulevard urbain à circulation réduite (une voie dans chaque sens et tous les carrefours aménagés en giratoires) avec le nouveau Busway en site propre sur la partie centrale, depuis la périphérie sud (Vertou) jusqu'au centre-ville (Cathédrale). Le Busway est à la fois louangé et critiqué par les Nantais, louangé comme nouveau Transport en commun en site propre (TCSP) à haute fiabilité, critiqué pour les lourdes perturbations apportées au trafic automobile pendant toute la durée des travaux et depuis, l'axe emprunté n'ayant pas de dérivation naturelle capable d'absorber les mêmes débits. C'est ce qui explique le choix du premier scénario stratégique, détaillé dans la section suivante. Les deux autres scénarios stratégiques correspondent à ce que serait la situation 2008 si le PDU avait été réduit à son strict minimum pour l'un (PDU Min), dans une perspective favorable à l'automobile, et à l'opposé si toutes les mesures envisagées dans le PDU avaient été réalisées pour l'autre (PDU Max), dans une perspective volontariste.

Les scénarios-tests permettent l'analyse de l'impact des différentes mesures de planification possibles en faisant varier une seule variable à la fois : la demande de déplacements, le nombre des déplacements en véhicules individuels (covoiturage), le prix des carburants, celui des parkings ou des transports en commun, la fréquence des TC, les limitations de vitesse.

Il n'est pas possible d'analyser ici dans le détail les résultats obtenus avec la «méthode EvalPDU » pour tous les scénarios et tests de sensibilité : trois scénarios sont d'abord présentés pour illustrer les impacts que la méthode des modélisations permet de mettre en relief, d'une part le scénario stratégique de la ligne 4 Busway (Section 3.4) et d'autre part les deux tests de sensibilité au taux de covoiturage et au nombre de déplacements (Section 3.5). Puis les résultats obtenus avec la «méthode simplifiée » sont comparés de manière synthétique pour l'ensemble des scénarios (Section 3.6) avant de revenir sur une ré-analyse méthodologique en conclusion (Section 4).

\subsection{Le scénario « sans Busway »}

Ce scénario est défini très simplement comme la situation 2008 sauf en ce qui concerne la mise en service du Busway : absence d'aménagement des boulevards de la pénétrante sud, maintien de leur caractère de pénétrante rectiligne (Figure 5), pas de TCSP et maintien des quatre lignes de bus 26 à 29. À l'inverse des autres scénarios, on évalue dans cette section l'impact de l'aménagement de la Ligne 4 Busway en comparant la situation de référence 2008 (avec Busway) à la situation simulée par le scénario « sans Busway ».

Le premier effet de la création de ce TCSP est de presque doubler le nombre de déplacements en transports en commun sur cet axe : 13000 voyages/jour sur les bus de l'ensemble des quatre lignes en 2003 pour 24000 en Busway en 2008. Le second effet est principalement lié à la forte réduction du trafic automobile sur l'axe de l'ancienne pénétrante transformée en boulevard urbain occupé par le TCSP en partie centrale (Figure 6). Aux heures de pointe elle peut atteindre près de $50 \%$ d'un trafic autrefois déjà très chargé, et elle génère d'une part d'importantes réductions de vitesses et d'autre part de forts reports de trafic soit vers des itinéraires de contournement locaux, plus ou moins parallèles et dirigés vers les autres lignes de ponts de la Loire, soit vers le périphérique sud-ouest et surtout vers le

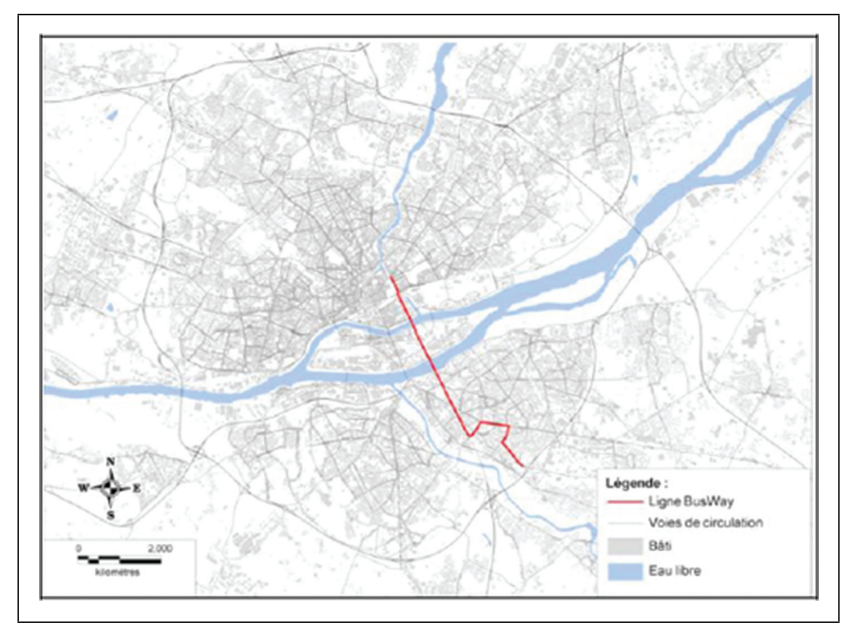

Fig. 5 Tracé de la Ligne 4 Busway 
(a)

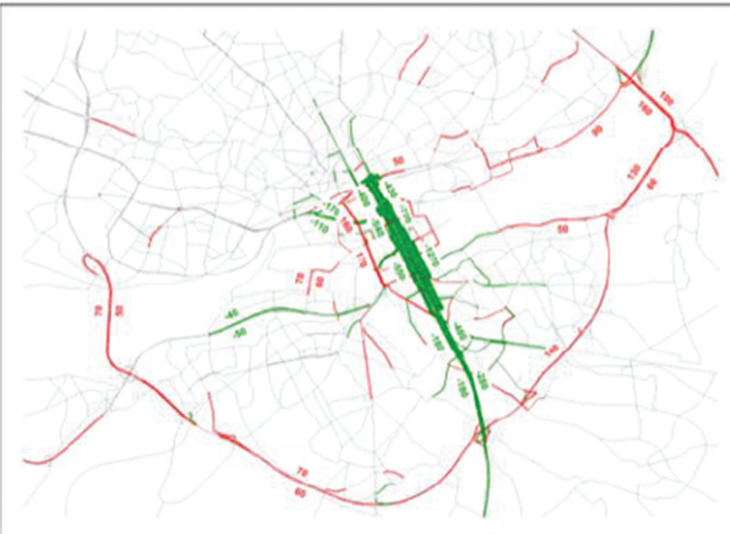

(b)

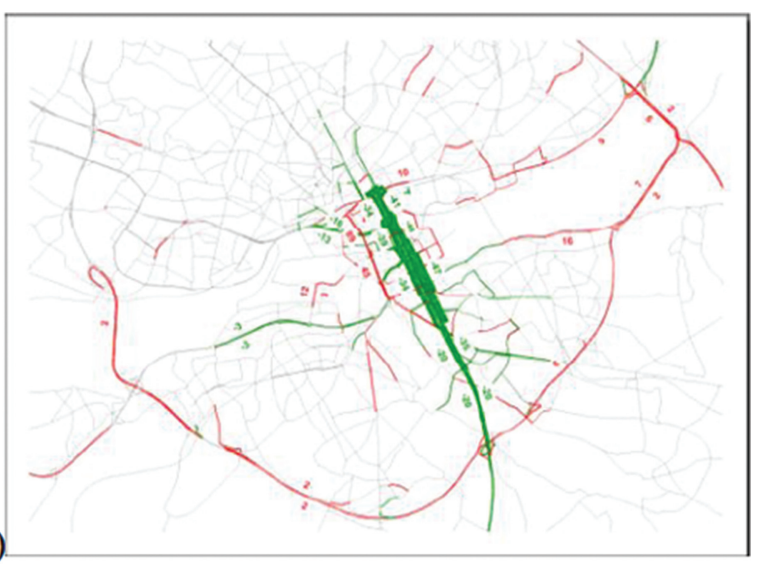

Fig. 6 Variations du trafic (heure de pointe du soir, jour ouvrable) induites par l'aménagement de la Ligne 4 Busway sur son parcours et sur les axes voisins du sud de l'agglomération, (a) en nombres de véhicules/heure, (b) en pourcentages du trafic 2008 (la largeur des tronçons est proportionnelle à la variation, en vert les diminutions, en rouge les augmentations)

périphérique sud-est avec des incidences notables sur les axes de pénétration de l'est vers le centre sur les rives de la Loire (Figure 6). On note de légères réductions de trafic sur les axes « diamétraux », c'est-à-dire en prolongement de la Ligne 4 vers le nord, probablement induites par des reports vers le périphérique. À l'échelle de l'ensemble de Nantes Métropole la diminution du nombre de déplacements en véhicules individuels induite par la création de la Ligne 4 est inférieure à $1 \%$, ce qui représente un impact net non significatif.

Les principaux impacts environnementaux apparaissent clairement sur les Figures 7 et 8 . Sur la cartographie des émissions (Figure 7) on retrouve les impacts directs des accroissements et diminutions de trafic, tronçon par tronçon mais avec quelques modulations selon le niveau de trafic dans la situation de référence. On retrouve ces mêmes variations sur les cartographies des concentrations de polluants (Figure 8), avec cependant des effets de dilution et d'étalement dus à la dispersion atmosphérique. Au plan local, sur l'axe du Busway on observe une diminution supérieure à $10 \%$ des émissions de NOx et une baisse des concentrations de $\mathrm{NO} 2$ pouvant dépasser $4 \mathrm{\mu gm}^{-3}$, soit environ $10 \%$ des concentrations de la situation de référence ; donc un impact local sans «perte en ligne ». En revanche une augmentation est observée en proximité de voirie sur les itinéraires de contournement, généralement

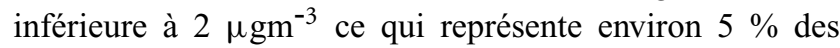
concentrations de référence. L'environnement de ces axes étant moins résidentiel, la population résidant dans une zone aux concentrations de $\mathrm{NO} 2$ supérieures à $40{\mu \mathrm{gm}^{-3}}^{-3}$ en moyenne annuelle diminue de 1300 habitants (-4 \%). Pour une analyse des reports de trafic et de leurs motivations on renvoie au rapport de Fouillé et Karsenty sur l'enquête menée dans le Busway [24, 39]. (a)

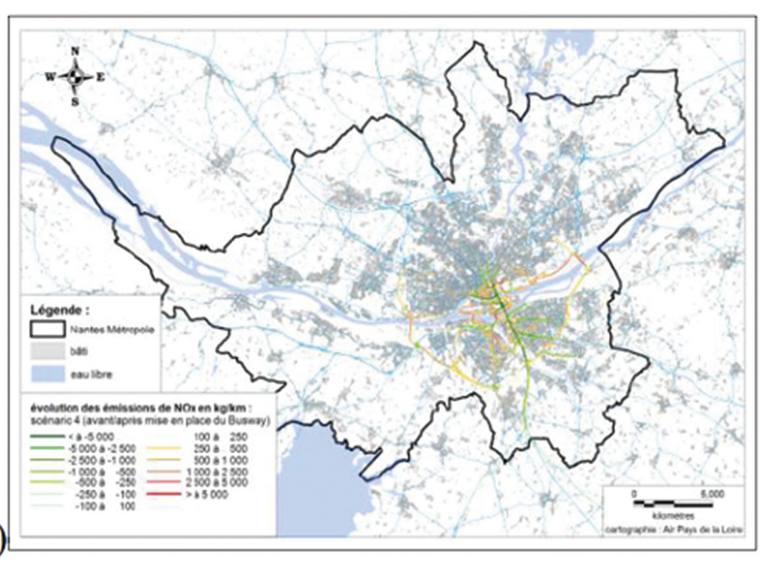

(b)

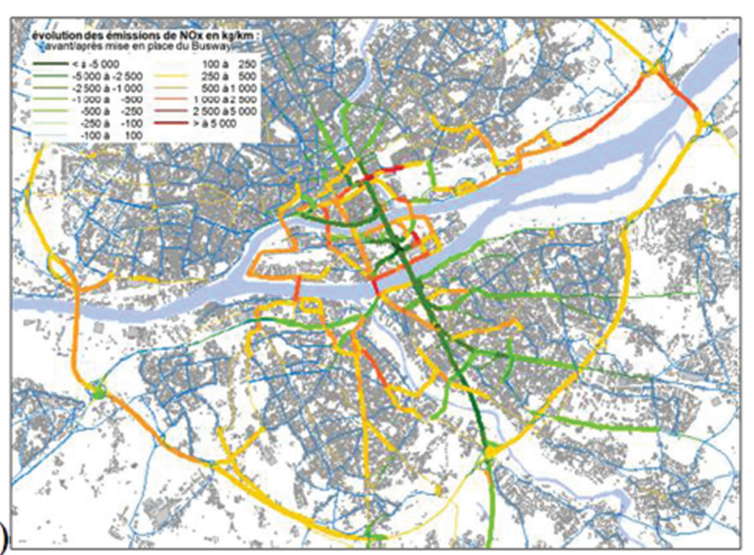

Fig. 7 Variations des émissions de NOx $(\mathrm{kg} / \mathrm{km})$ induites par la mise en œuvre de la ligne 4 Busway, (a) sur Nantes Métropole, (b) sur la partie sud de Nantes 

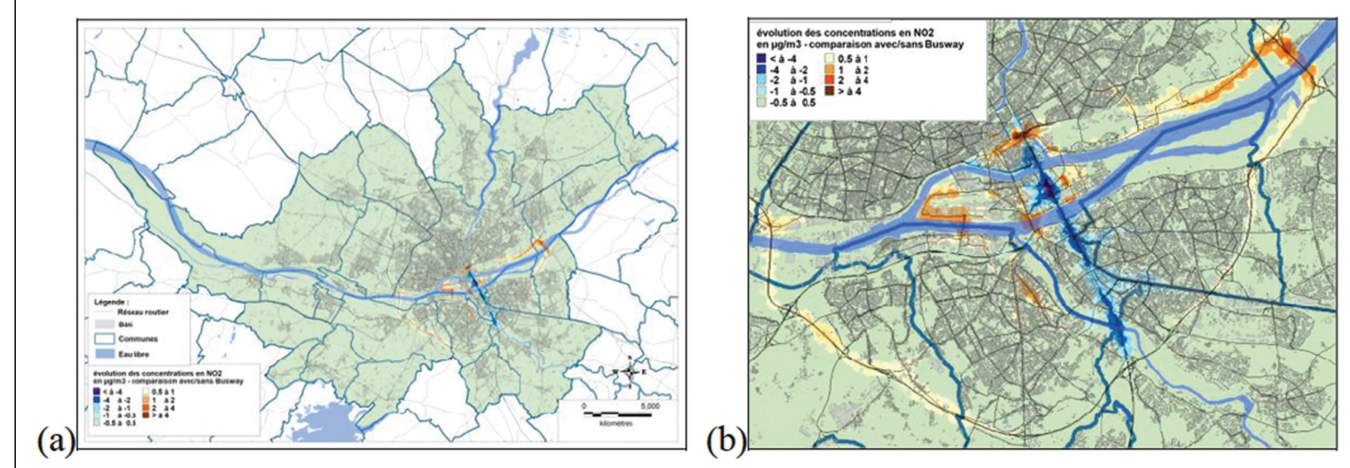

Fig. 8 Variations des concentrations de $\mathrm{NO}_{2}(\mu \mathrm{g} / \mathrm{m} 3)$ induites par la mise en œuvre de la ligne 4 Busway, (a) sur Nantes Métropole, (b) sur la partie sud de Nantes

\subsection{Les scénarios « Covoiturage » et «Mobilité »}

Ces deux scénarios sont présentés ensemble car ils représentent des tests de sensibilité presque symétriques en termes de variables d'analyse mais dont les impacts ne sont pas tout à fait symétriques. Ils reposent tous les deux uniquement sur le nombre de déplacements, le premier uniquement sur les déplacements en véhicules individuels, le second sur la demande globale de mobilité, sans aucune modification des offres (réseau routier et réseau TC). Le scénario «Covoiturage » suppose que $33 \%$ des déplacements en véhicule individuel sont remplacés par du covoiturage, soit une diminution de $25 \%$ du trafic automobile en véhicule léger, sans modification de la demande de transports en commun. Dans le scénario «Mobilité » le nombre de déplacements par personne et par jour est augmenté de $20 \%$, représentant une demande sociétale accrue, ici aussi sans changement de l'offre. Dans les deux cas les trafics d'automobiles individuelles, mais aussi de poids lourds et de transports en commun routiers, s'adaptent aux nouvelles conditions de circulation (vitesses et saturations) sur le réseau routier. Les variations des valeurs des variables d'entrée sont volontairement choisies fortes pour mettre en lumière les impacts de second ordre ou de rétroaction.

La Figure 9 illustre l'effet de ces deux scénarios sur le trafic routier. Dans le scénario "Covoiturage » on observe une très forte diminution de la congestion, avec

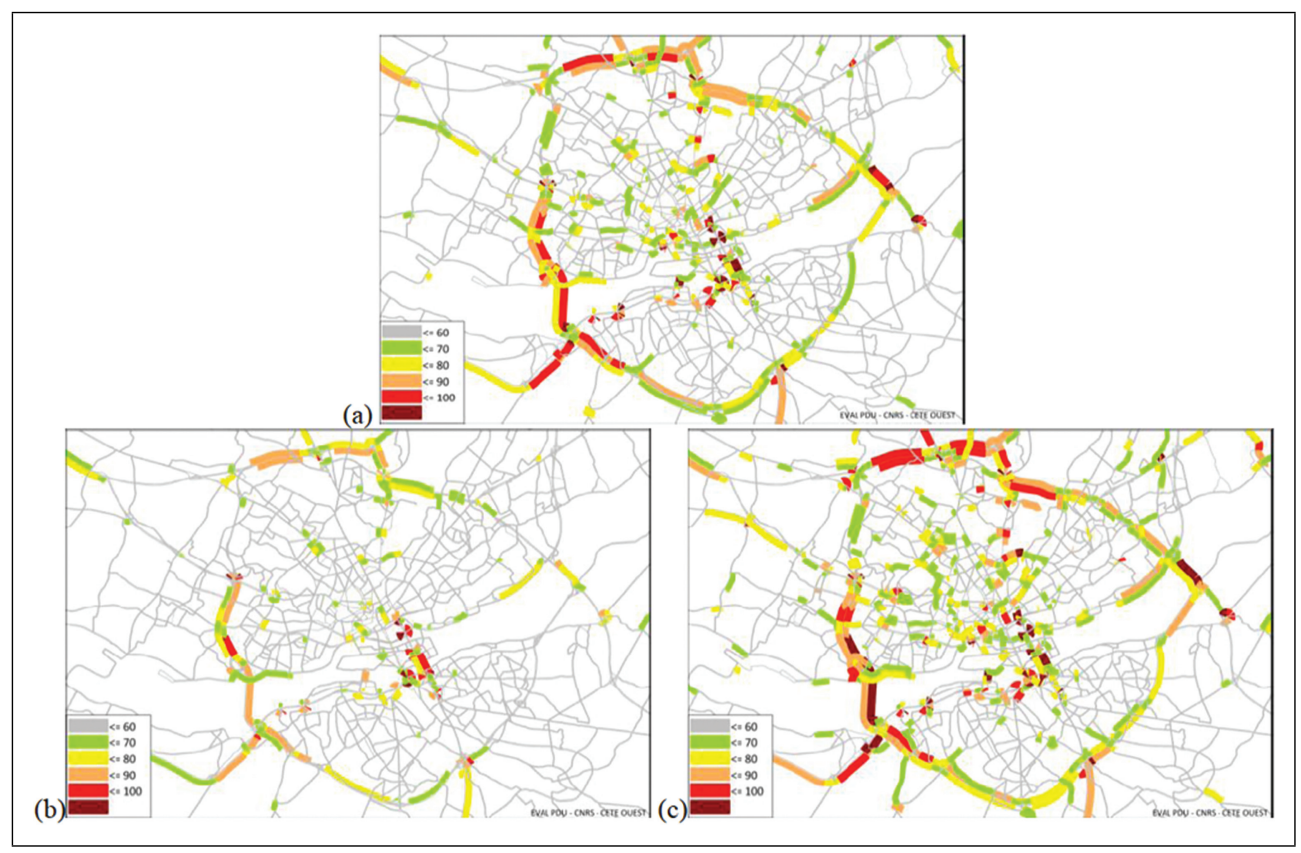

Fig. 9 Niveau de saturation (en \%) des tronçons routiers à l'heure de pointe du matin dans la situation de référence 2008 (a) et selon les scénarios « Covoiturage » (b) et « Mobilité » (c) 
une quasi-disparition des saturations dans le centre sauf au passage des ponts : le nombre de sections saturées à plus de $90 \%$ passe en effet de 76 à 31 en heures de pointe du matin et de 75 à 29 en heures de pointe du soir. Le nombre de véhicules.kilomètres est réduit d'environ $23 \%$, ce qui signifie que les distances parcourues augmentent très légèrement (puisque le nombre de véhicules est réduit de $25 \%$ ), les voiries rapides moins saturées devenant plus attractives. Dans la réalité cette diminution de la congestion rendant l'automobile plus attractive induirait probablement une rétroaction (de second ordre) de report du transport en commun vers l'automobile. Dans le scénario « Mobilité » $(+20 \%)$ le nombre de sections saturées à plus de $90 \%$ passe de 76 à 106 en heure de pointe du matin, principalement sur le périphérique mais aussi sur de très nombreux tronçons (ou carrefours) dans le cœur de la ville. On observe une augmentation de $+16 \%$ des déplacements en véhicules automobiles à l'intérieur de Nantes et de +18 à $20 \%$ dans le reste du domaine. Cependant la part modale des TC augmente d'environ un point, du fait de l'aspect dissuasif des congestions du réseau routier.

Les impacts environnementaux sont illustrés par le Tableau 1 et la Figure 10 pour les consommations d'énergie, émissions de GES et de polluants atmosphériques, le Tableau 2 et la Figure 11 pour le bruit. Ces résultats illustrent les effets de plasticité et d'inertie du système de déplacements et les effets de non-linéarité des impacts environnementaux.

Dans le Tableau 1 observons d'abord la dernière ligne qui marque les effets d'un accroissement général de la demande de mobilité de $20 \%$. Du fait des reports modaux induits par l'accentuation des phénomènes de

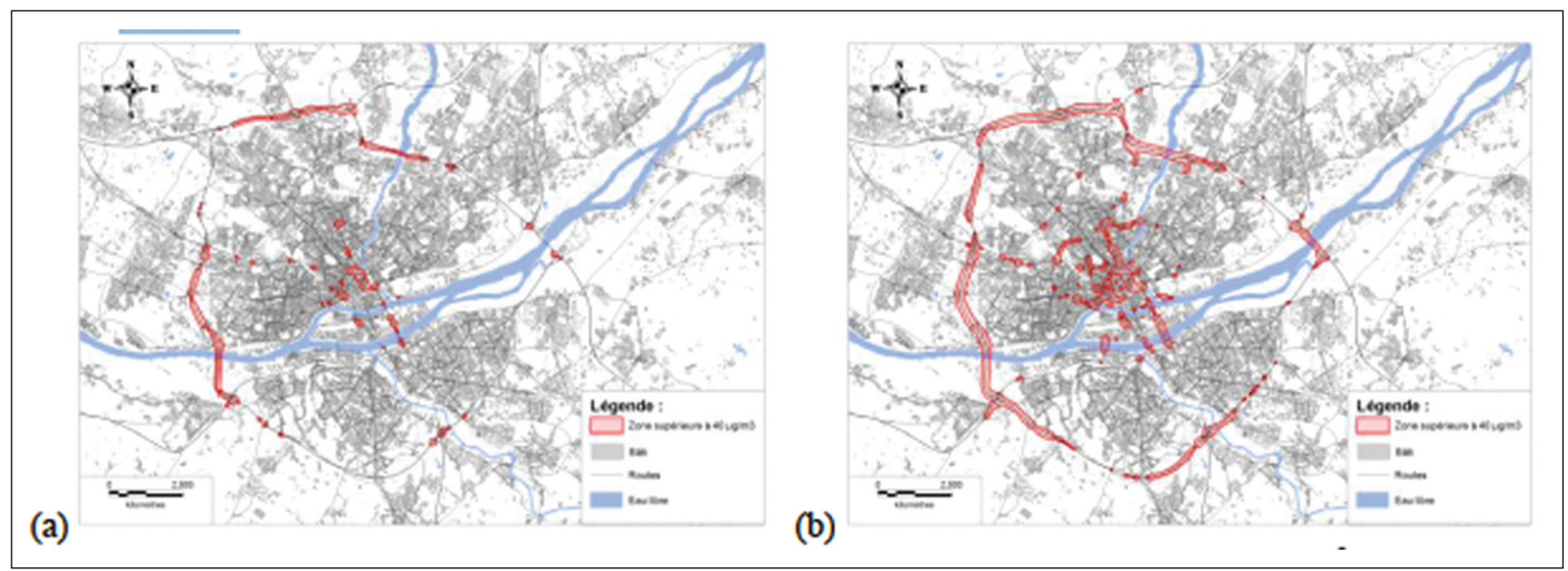

Fig. 10 Zones où les concentrations moyennes annuelles de $\mathrm{NO}_{2}$ dépassent le seuil de $40 \mu \mathrm{g} \mathrm{m}^{-3}$, selon les scénarios « Covoiturage » (a) et « Mobilité » (b)

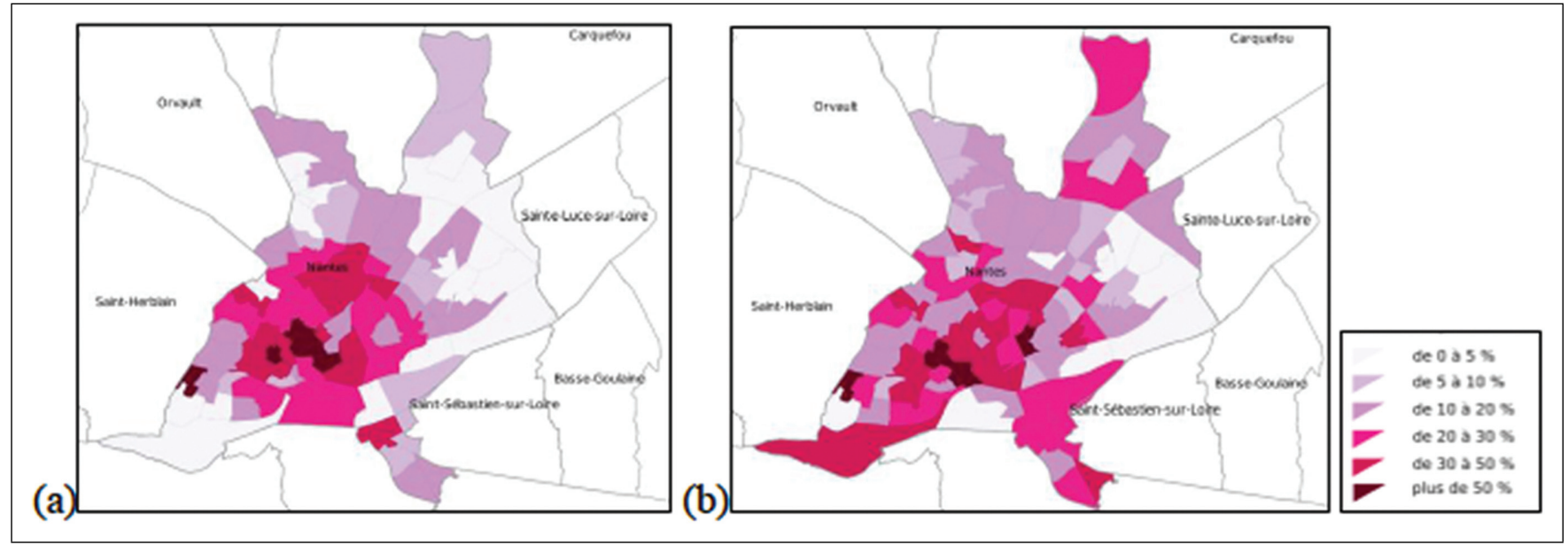

Fig. 11 Pourcentage d'habitants soumis à un dépassement du seuil de $68 \mathrm{~dB}$ dans chaque IRIS de la commune de Nantes, selon les scénarios « Covoiturage » (a) et « Mobilité » (b) [30, 31] 


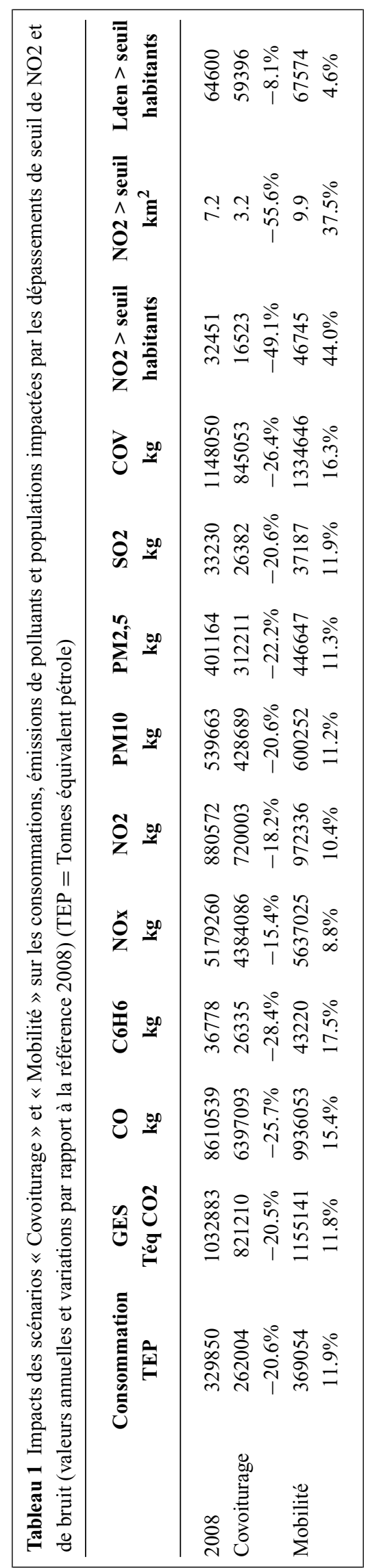

Necplus 


\begin{tabular}{|c|c|c|c|c|c|c|c|c|}
\hline \multirow[b]{2}{*}{ Lden Population } & \multicolumn{2}{|c|}{ Référence 2008} & \multicolumn{3}{|c|}{ Scénario 《 covoiturage » } & \multicolumn{3}{|c|}{ Scénario 《mobilité » } \\
\hline & Nombre & $\%$ & Nombre & $\mathbf{R} \%$ & D\% & Nombre & $\mathbf{R} \%$ & D\% \\
\hline$<50 \mathrm{db}$ & 67180 & 23,9 & 69628 & 24,8 & $+3,6$ & 66929 & 23,8 & $-0,4$ \\
\hline $50-55 \mathrm{db}$ & 29071 & 10,3 & 28764 & 10,2 & $-1,1$ & 27986 & 10,0 & $-3,7$ \\
\hline $55-60 \mathrm{db}$ & 33347 & 11,9 & 34636 & 12,3 & $+3,9$ & 33070 & 11,8 & $-0,8$ \\
\hline $60-65 \mathrm{db}$ & 54093 & 19,3 & 57052 & 20,3 & $+5,5$ & 51512 & 18,3 & $-4,8$ \\
\hline $65-70 \mathrm{db}$ & 53505 & 19,0 & 50807 & 18,1 & $-5,0$ & 55640 & 19,8 & $+4,0$ \\
\hline $70-75 \mathrm{db}$ & 36243 & 12,9 & 33254 & 11,8 & $-8,2$ & 38092 & 13,5 & $+5,1$ \\
\hline$>75 \mathrm{db}$ & 7478 & 2,6 & 6776 & 2,4 & $-9,4$ & 7688 & 2,7 & $+2,8$ \\
\hline
\end{tabular}

congestion il se traduit par une croissance globale du trafic automobile de seulement $17 \%$, la part modale des transports en commun augmentant d'environ un point. Cependant les consommations de carburant et émissions de GES n'augmentent que de moins de $12 \%$ et les évolutions des émissions de polluants atmosphériques sont assez variables, de l'ordre de $17 \%$ pour le benzène et les COV mais moins de $9 \%$ pour les NOx.

Dans le scénario « Covoiturage » la réduction du nombre de véhicules-kilomètres de $23 \%$ se traduit par une réduction de seulement $20,5 \%$ de la consommation de carburant et des émissions de GES, mais aussi par des réductions nettement plus fortes des émissions de benzène, $\mathrm{CO}$ et $\mathrm{COV}$, liées aux différences de condition de circulation, plus proches des vitesses limites autorisées.

Le scénario «Mobilité » illustre bien la non-linéarité du système : augmentation de $20 \%$ de la demande, de $16-18 \%$ du nombre de déplacements en automobiles, de $12 \%$ des consommations et émissions de GES, de $9 \%$ pour les NOx (mais $17 \%$ pour le benzène et les COV $\mathrm{du}$ fait des embouteillages), et augmentation de $44 \%$ du nombre d'habitants soumis aux plus fortes concentrations de polluants.

L'impact des scénarios sur les pollutions les plus fortes est évalué par l'identification des zones soumises à une concentration de $\mathrm{NO} 2$ dépassant le seuil réglementaire de $40 \mu \mathrm{g} \mathrm{m}^{-3}$ en moyenne annuelle (Figure 10), ce qui permet d'obtenir leur surface totale par intégration spatiale (Tableau 1). L'algorithme de répartition des habitants par bâtiment dans chaque IRIS mis en place dans OrbisGIS permet de déterminer le nombre d'habitants des bâtiments implantés dans ces zones impactées et au total le nombre d'habitants concernés (Tableau 1). Du fait de la forte disparité des densités de population en centre-ville et autour du périphérique, on observe des variations à peu près symétriques du nombre d'habitants impactés alors que les surfaces impactées présentent de fortes différences.

Les niveaux de bruit moyen journaliers $\left(\mathrm{L}_{\mathrm{den}}\right)$ générés par les réseaux routier et de transports en commun, calculés au sol et sur chaque façade des bâtiments de l'agglomération [30], croisés avec les répartitions des habitants par bâtiment, permettent de déterminer le nombre total d'habitants soumis à un niveau de bruit donné. Le Tableau 2 les présente par classes de niveau de bruit. On voit que l'impact de ces deux scénarios reste faible à l'échelle de l'agglomération, malgré les fortes variations imposées aux variables d'entrée, avec un glissement d'une classe vers l'autre généralement inférieur à $1 \%$ de la population, vers les classes de plus faible bruit dans le scénario "Covoiturage" et vers les classes de bruit plus fort dans le scénario "Mobilité".

Cependant ces faibles variations à l'échelle de l'ensemble du territoire ne doivent pas cacher qu'il existe des variations d'impacts non négligeables à l'échelle locale. À une résolution permettant de distinguer les immeubles les cartes détaillées sont d'une grande utilité pour distinguer les « points noirs » qui peuvent être créés par un scénario ou au contraire améliorés par un autre [31]. Par contre à une échelle moins fine permettant de couvrir l'ensemble de l'agglomération elles deviennent difficiles à analyser car les détails ne sont plus visibles. C'est pourquoi la Figure 11 présente des résultats d'échelle intermédiaire : dans chaque IRIS le nombre d'habitants soumis à un niveau de bruit moyen journalier supérieur au seuil de $68 \mathrm{~dB}$ fixé par le décret n ${ }^{\circ}$ 2006-361 du 24 mars 2006 relatif à l'établissement des cartes de bruit et des plans de prévention du bruit dans l'environnement (les zones blanches ne contiennent aucun habitant soumis au dépassement de seuil). On note en particulier des variations significatives de la population impactée dans les secteurs nord-est de la commune. 


\begin{tabular}{|c|c|c|c|}
\hline Nom & Variable & Valeur & Étapes de modélisation \\
\hline Covoiturage & $\begin{array}{l}\text { Nombre moyen de } \\
\text { passagers/véhicule }\end{array}$ & $+33 \%$ & $\mathrm{~A}+\mathrm{E}+\mathrm{Di}$ \\
\hline Mobilité & Nombre de déplacements & $+20 \%$ & $\mathrm{R}+\mathrm{A}+\mathrm{E}+\mathrm{Di}$ \\
\hline Carburant & Coût du carburant autos & $+100 \%$ & $\mathrm{D}+\mathrm{R}+\mathrm{A}$ \\
\hline Sans Busway & Lignes TC, TCSP & $-\mathrm{Li} 4,+\mathrm{Li} 26-29$ & $\mathrm{D}+\mathrm{R}+\mathrm{A}+\mathrm{E}+\mathrm{Di}$ \\
\hline PDU Min & Réseaux routier et $\mathrm{TC}$ & TC 2002, Ponts & $\mathrm{D}+\mathrm{R}+\mathrm{A}+\mathrm{E}+\mathrm{Di}$ \\
\hline PDU Max & Réseau TC & $\begin{array}{l}\text { Tram-train, Tram }+ \text {, Busway }+ \text {, tarifs } \\
\text { TC/2 }\end{array}$ & $\mathrm{D}+\mathrm{R}+\mathrm{A}+\mathrm{E}+\mathrm{Di}$ \\
\hline Stationnement & Pénalité de stationnement (temps) & $+100 \%$ & $\mathrm{R}+\mathrm{A}$ \\
\hline Fréquence TC & Fréquence TC & $+30 \mathrm{ou}+50 \%$ & $\mathrm{R}$ \\
\hline Tarif TC & Tarifs tickets et abonnements & $-50 \%$ & $\mathrm{R}+\mathrm{A}$ \\
\hline \multirow[t]{2}{*}{ Vitesses limites } & $\begin{array}{l}\text { Vitesses limites du centre-ville et du } \\
\text { périphérique }\end{array}$ & $50 \rightarrow 30 \mathrm{~km} / \mathrm{h}$ & $\mathrm{D}+\mathrm{R}+\mathrm{A}+\mathrm{E}+\mathrm{Di}$ \\
\hline & & $90 \rightarrow 70 \mathrm{~km} / \mathrm{h}$ & \\
\hline
\end{tabular}

\subsection{Impacts des scénarios, approche comparative et synthétique}

Les scénarios «Covoiturage », « Mobilité » et « Busway » ayant été définis plus haut, on présente ici les définitions des autres scénarios (Tableau 3), leurs conséquences sur le trafic routier et la qualité de l'air, puis leurs impacts à l'échelle de l'agglomération à partir d'indicateurs agrégés.

Le scénario «PDU Min » est défini par le réseau de transports en commun de 2002 sans nouvel aménagement, et le réseau routier de 2008 avec ses nouveaux aménagements plus, dans une perspective favorable aux déplacements automobiles, la mise en service avancée de la nouvelle ligne de ponts de l'est de l'Ile de Nantes (Tabarly et Senghor). Dans ce scénario la part modale de la voiture se consolide (+ 0,3 point), le nombre de sections saturées à plus de 90 $\%$ décroît de 76 à 60, parce que les TC n'apportent pas de nouvelle contrainte à l'espace dédié aux voitures. Les distances parcourues diminuent très légèrement du fait que l'itinéraire le plus rapide correspond plus souvent au trajet le plus direct. Ce scénario a un impact moyen sur la qualité de l'air peu perceptible à l'échelle de l'agglomération (inférieur à $1 \%$ ) mais un impact local très contrasté : une augmentation relativement importante des concentrations sur l'île de Nantes et le boulevard de Vendée, et une légère baisse sur les itinéraires de contournement (pont de Bellevue, périphérique sud et ouest). Malgré une réduction de $-4 \%$ de la surface présentant des concentrations de NO2 supérieures à $40 \mathrm{\mu gm}^{-3}$, ce scénario augmente de $+6 \%$ la population impactée (34 486 habitants) car l'augmentation des concentrations en centre-ville présente un impact plus important que la baisse des concentrations en périphérie.

Le scénario de PDU volontariste «PDU Max » reprend tous les aménagements envisagés dans le plan de $2002: 1$. prolongement de la Ligne 1 est (Beaujoire-Carquefou), 2. aménagement complet (diamétral) de la Ligne 4 Busway de Vertou jusqu'au Cardo, 3. création de la ligne 5 (Baco-Chantenay), 4. réalisation de la jonction nord des lignes de tram 1 et 2,5 . réalisation de la liaison tram-train au nord jusqu'à La Chapelle-sur-Erdre et Carquefou, 6. division par deux des tarifs TC. Le renforcement marqué de l'offre TC se traduit localement par une certaine réduction de la capacité automobile. La part modale TC gagne près de 4 points, celle de la voiture est globalement en recul de plus de 5 points, les distances parcourues diminuant de $3 \%$. Le nombre de sections saturées à plus de $90 \%$ baisse de 76 à 64 . On observe une réduction de $16 \%$ des déplacements automobiles sur les trajets internes à Nantes, avec des vitesses réduites de $11 \%$, et de 1 à $3 \%$ sur les autres trajets. Ce scénario a un impact sur les émissions et consommations du secteur routier assez homogène à l'échelle de l'Agglomération, avec des réductions comprises entre 3 et $5 \%$. Les polluants présentant l'effet le plus marqué sont le $\mathrm{CO}$, le $\mathrm{C} 6 \mathrm{H} 6$ et les $\mathrm{COV}$. Une baisse des émissions de NOx entre $-2,5$ et $-5 \%$ est observée dans le domaine intra-périphérique, alors que les émissions extra-périphériques sont globalement similaires à celles de la situation de référence. En centre-ville, cette diminution est plus marquée et peut atteindre $-25 \%$. Ce scénario permet de diminuer de $-15 \%$ la surface présentant des 
concentrations de $\mathrm{NO} 2$ supérieures au seuil de $40 \mathrm{\mu gm}^{-3}$ et de $-28 \%$ la population résidante exposée à ce dépassement en moyenne annuelle (23 442 habitants).

Le scénario «Carburant » est défini par le doublement $\mathrm{du}$ prix moyen des carburants, de 1,25 à $2,50 €$ par litre, qui pénalise le choix modal du véhicule individuel (et les transports de marchandises en véhicules légers et poids lourds). On observe une chute du trafic d'environ $4 \%$, le bénéfice étant réparti entre la marche et les TC, une réduction des déplacements en véhicules individuels négligeable à l'intérieur de la métropole, entre 0 et $-3 \%$, et de -9 à $-14 \%$ dans le reste du domaine, accompagnée d'une légère augmentation des vitesses moyennes $(+2 \%)$. En HPM, on comptabilise une dizaine de sections passant sous la barre des $90 \%$ de saturation. L'augmentation du prix du carburant entraîne une réduction homogène des émissions et consommations d'énergie entre 3 et $4 \%$ à l'échelle de l'agglomération, mais elle a un impact hétérogène sur les émissions de NOx : si la majorité des axes routiers présente une légère diminution des émissions, inférieure à $-5 \%$, quelques axes montrent une augmentation plus ou moins marquée, de $+2,5 \%$ jusqu'à plus de $+50 \%$, en relation avec des augmentations locales de trafic. L'impact sur les concentrations annuelles de $\mathrm{NO} 2$ est faible, globalement inférieur à $1 \mu \mathrm{gm}^{-3}$ et limité au périphérique et quelques boulevards. Il a peu d'effet sur la population résidente concernée par les dépassements du seuil de $40 \mu \mathrm{gm}^{3}(-3 \%)$. Il a un effet très minime sur le bruit, le nombre d'habitants soumis à un Lden $>68 \mathrm{~dB}$ diminuant de 433 , soit moins de $1 \%$.

Le scénario «Stationnement » est défini par le doublement des pénalités de stationnement dû soit au doublement du temps de recherche généré par une réduction $\mathrm{du}$ nombre de places de parking, soit au doublement $\mathrm{du}$ tarif du stationnement, plus l'extension des zones de stationnement payant réalisée en réalité après 2008. Ce doublement des pénalités de stationnement provoque une chute de la part modale des automobiles de 5,5 points en intra-Nantes (soit près de $9 \%$ ), au bénéfice de la marche et des TC. Sur les autres trajets internes à Nantes Métropole la baisse de la part modale des véhicules individuels est très faible, le bénéfice se portant plutôt sur les TC.

Les scénarios «Fréquences TC » et «Tarifs TC » correspondent respectivement à une hausse de 30 à $50 \%$ de la fréquence des TC (en pratique ce sont les temps d'attente qui sont divisés par 2) et à la division par 2 des tarifs TC. Les parts modales et les conditions de trafic sont assez peu sensibles à l'augmentation des fréquences des transports en commun urbains, du fait qu'elles sont déjà relativement élevées dans la situation de référence, notamment sur les axes lourds. La légère réduction du temps d'attente qui en découle n'apporte donc qu'un faible gain relatif de temps de parcours. Par contre la baisse des tarifs apporte un gain sensible de part modale TC, de l'ordre de 1,5 points, au détriment de la voiture (-1 point) et de la marche à pied $(-0,5$ point). La réduction du nombre de voies saturées à plus de $90 \%$ en heure de pointe reste néanmoins faible, de 76 à 70 .

Le scénario «Vitesses limites » est défini par la réduction des vitesses limites urbaines, de 50 à $30 \mathrm{~km} / \mathrm{h}$ sur l'ensemble du centre ville et de 90 à $70 \mathrm{~km} / \mathrm{h}$ sur le périphérique. L'impact de cette réduction sur les parts modales et le volume de la circulation automobile est très faible. On observe cependant une réduction des distances parcourues (environ $2 \%$ ) probablement due au fait que les voiries rapides, qui allient souvent un gain de temps à l'allongement de parcours, deviennent moins attractives avec des vitesses autorisées réduites de $20 \mathrm{~km} / \mathrm{h}$. Le nombre de sections saturées en heures de pointe est inchangé. En termes d'émissions de polluants et de consommation énergétique, à l'échelle de l'Agglomération l'impact de ce scénario est peu perceptible et globalement inférieur à $1 \%$. Les concentrations annuelles varient de $-3.5 \mu \mathrm{g} \mathrm{m}^{-3}$ (périphérique sud) à $+3 \mu \mathrm{g} \mathrm{m}^{-3}$ (centre-ville) par rapport à la situation de référence $(-8 \%$ à $+15 \%)$. Les branches sud et ouest du périphérique présentent une baisse des concentrations liée à la réduction des émissions ; à l'inverse, on note une augmentation des concentrations de $1,5 \mu \mathrm{gm}^{-3}$ au niveau de certains grands boulevards. En centre-ville, la réduction de vitesse à $30 \mathrm{~km} / \mathrm{h}$ entraîne une augmentation des concentrations de $\mathrm{NO} 2$, en lien avec une augmentation des émissions aux faibles vitesses. La réduction de vitesse au niveau du périphérique permet de diminuer de $-9 \%$ la surface présentant des concentrations supérieures à 40 $\mu \mathrm{gm}^{-3}$ mais l'effet reste très faible en termes de population soumise à ces concentrations $(-2 \%)$.

Parallèlement aux indicateurs obtenus par la « méthode simplifiée » estimés pour l'ensemble du territoire de Nantes Métropole, à partir des simulations des consommations et des émissions détaillées sur l'ensemble du territoire on a également calculé des intégrales sur l'ensemble de l'année, puis on les a normalisées par les valeurs obtenues pour la situation de référence. Les indices normalisés obtenus par ces deux méthodes sont comparés sur les Figures 12 et 13. On voit que les variations des indices des mêmes indicateurs sont très comparables pour les deux méthodes. On remarque que les variations des indices des deux premiers scénarios sont presque un ordre de grandeur supérieures à celles des autres scénarios, ce qui signifie que les mesures envisageables pour un PDU sont du même ordre d'influence que des variations du nombre de déplacements, notamment en véhicules individuels, de l'ordre de 2 à $3 \%$ seulement.

On note que les indices normalisés d'émissions sont pratiquement identiques pour les différents polluants réglementés, pour chaque scénario, sauf pour le scénario de réduction des vitesses limites. De même les indices 

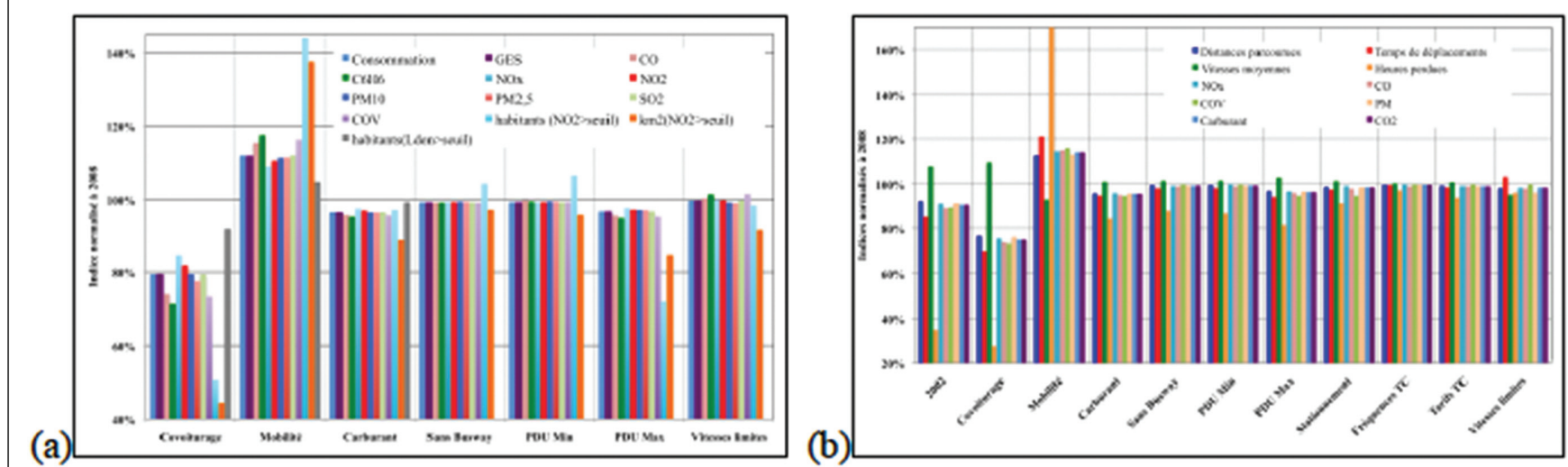

Fig. 12 Indices représentatifs des impacts environnementaux des scénarios: (a) intégrales sur Nantes Métropole des consommations et émissions annuelles normalisées par celles de la situation 2008, (b) calculs simplifiés sur une journée ouvrable
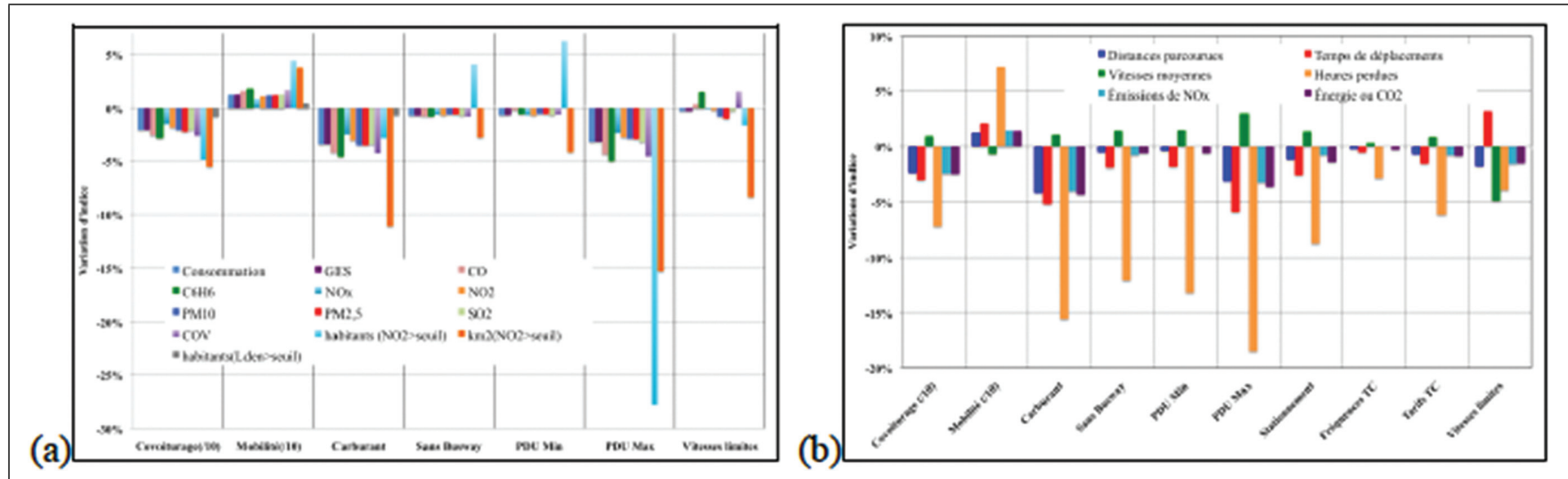

Fig. 13 Variation par rapport à la situation 2008 des indices représentatifs des impacts environnementaux des scénarios: (a) intégrales sur Nantes Métropole des consommations et émissions annuelles, (b) calculs simplifiés sur une journée ouvrable

de consommation d'énergie (carburant) et d'émission de GES (CO2) sont identiques dans la Figure 12b puisque ces deux indicateurs sont directement proportionnels dans la « méthode simplifiée », mais aussi presque identiques dans les résultats de simulations détaillées (Figure 11a), ce qui valide cette hypothèse de simplification. Dans la Figure $13 \mathrm{~b}$, pour faciliter la lecture on a retiré la situation 2002 , conservé un seul indice pour les émissions de polluants (NOx), et un seul pour la consommation d'énergie/carburant et pour l'émission de GES/CO2 ; enfin on a divisé par 10 les variations d'indices des deux premiers scénarios.

Le scénario de réduction des vitesses limites n'est pas véritablement comparable avec les autres (Figure 13b), car dans ce cas la baisse de vitesse s'accompagne d'une baisse de la congestion. Le temps total augmente mais moins d'heures sont perdues du fait de la congestion - en réalité elles sont alors perdues du fait de la réglementation mais l'indicateur ne saisit pas cette subtilité puisqu'il calcule la perte de temps liée aux écarts par rapport à la vitesse réglementaire: en abaissant la vitesse autorisée on limite les pertes de vitesses et le point de saturation de la voirie n'est jamais atteint (sauf débit extrême). D'autre part, en abaissant la vitesse du réseau routier, et surtout celle du périphérique, on rend plus attractifs les itinéraires les plus courts, ce qui explique la réduction des distances parcourues (en plus d'une réduction de la part modale). Cette baisse de trafic génère une réduction de la consommation de carburant, et des émissions de NOx et $\mathrm{CO}$ dans une proportion similaire. Ce qui est plus surprenant avec cette mesure, c'est qu'elle génère une baisse plus importante des émissions de particules, mais aussi une hausse des émissions de COV. C'est le seul scénario pour lequel les émissions prennent des directions aussi différenciées.

Le scénario «Carburant» de multiplication par deux des prix du carburant automobile, dont les impacts paraissent relativement faibles dans les simulations détaillées, se révèle ici induire des variations des indicateurs du même ordre que le scénario de PDU volontariste « PDU Max ». La 
comparaison entre ces deux scénarios est particulièrement intéressante. D'un côté une politique volontariste composée d'un ensemble d'actions en faveur des transports en commun, de dissuasion du stationnement en centre-ville, etc. et de l'autre l'action mécanique du doublement du prix du carburant. Ces deux scénarios agissent dans des proportions comparables, mais les nuances valent d'être relevées. Étonnamment, les effets observés avec «PDU Max » peuvent s'apparenter à ceux d'une bonne politique de fluidification du trafic (a priori pro-automobile) là où l'action par les prix s'apparente davantage dans ses effets à une action pro-environnementale. En effet, « PDU Max » améliore les vitesses et diminue les durées de conduite et les temps perdus plus que ne le fait la hausse du prix des carburants. En revanche, l'action par les prix diminue plus les distances parcourues, les consommations de carburant et les émissions de polluants atmosphériques. Ces conclusions doivent cependant être relativisées du fait de la proximité des résultats obtenus et des incertitudes du mode de calcul des indicateurs.

Les variations des indicateurs normalisés sont également étrangement semblables pour les scénarios «PDU Min » et «Sans Busway », autant à partir des intégrales des simulations (Figure 13a) que pour les calculs simplifiés (Figure 12b) : (1) de faibles réductions des temps de déplacements et augmentations des vitesses de circulation, (2) de nettes diminutions des heures perdues, et (3) des variations négligeables des autres indicateurs. On note cependant une particularité observée pour ces deux scénarios seulement : une diminution des surfaces soumises aux concentrations élevées de $\mathrm{NO} 2$ accompagnée d'une augmentation du nombre d'habitants impactés. Cette ressemblance des indicateurs intégrés peut cependant cacher des impacts locaux relativement différents, ce que révèle la comparaison de la Figure 14 aux Figures $7 \mathrm{~b}$ et $8 \mathrm{~b}$ (celles-ci vues en inversant augmentations et diminutions). On retrouve bien sûr la forte influence de la pénétrante sud (non aménagée dans les deux cas), encore plus engorgée que dans la situation de référence, et une distribution complexe des axes « améliorés » et « dégradés » se compensant pour donner ces variations intégrales très faibles, ainsi qu'une tendance à l'aggravation de la circulation en centre-ville et une amélioration sur le périphérique qui génère cette évolution en sens inverses des surfaces et des nombres d'habitants soumis aux concentrations de NO2 supérieures à la valeur limite. Par contre on observe dans les deux situations de nettes différences dans l'enchevêtrement des axes en amélioration et en aggravation dans la zone intra-périphérique, avec par exemple une forte induction de trafic liée à la création de la nouvelle ligne de ponts Tabarly et Senghor, sur l'est de l'Ile de Nantes et le nord de Saint-Sébastien.

Pour terminer cette section on doit rappeler quelques incertitudes liées à la méthodologie des scénarios ou à sa mise en œuvre. La plus importante est celle de l'hypothèse de base « toutes choses égales par ailleurs » qui peut créer des irréalismes, notamment lorsqu'on impose des variations très fortes d'une seule variable. La seconde est l'absence ou l'insuffisance de prise en compte des rétroactions lorsqu'on ne met pas en œuvre la totalité des étapes de modélisation ou lorsqu'on utilise la « méthode simplifiée ». Nous avons essayé de minimiser ces deux

(a)
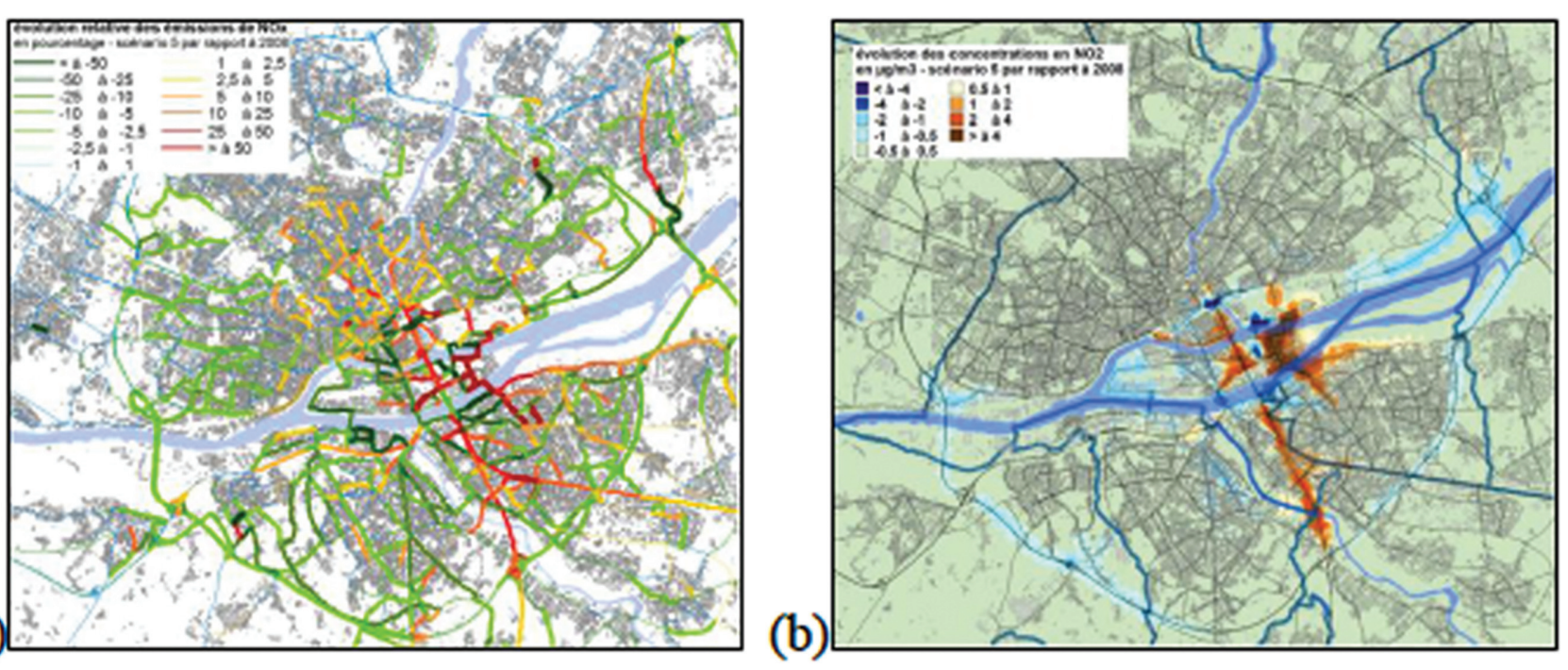

Fig. 14 Impacts du scénario «PDU Min » : (a) variations relatives des émissions de NOx (en \% par rapport à la situation 2008), (b) variations absolues des concentrations (en $\mu \mathrm{g} \mathrm{m}^{-3}$ ) 
facteurs d'incertitude par nos analyses préalables des étapes de modélisation nécessaires à chaque scénario, mais des doutes peuvent toujours subsister. Enfin la méthode analyse essentiellement des évolutions de relativement court terme pour lesquelles la répartition géographique des zones résidentielles et des activités peut être extrapolée ou supposée connue. Elle est moins bien adaptée aux changements de moyen et long termes, surtout si ces changements traduisent des interactions fortes entre déplacements et localisation, via notamment l'évolution du foncier, pour lesquelles il faudrait agrandir considérablement la chaîne de modélisations.

\section{Conclusion :}

\section{pour l'évaluation environnementale des futurs PDU, les modèles seront-ils adaptés ?}

La chaîne de modèles développée et perfectionnée dans le projet Eval-PDU est apte à analyser les impacts environnementaux d'un grand nombre d'actions sur la mobilité et les transports urbains. Le travail mené a confirmé cette capacité - déjà mise en évidence en partie par des études similaires en France et à l'étranger [15]. Il a montré qu'il était possible de produire une information utile pour l'évaluation ex-post ou ex-ante, sous forme d'indicateurs globaux ou statistiques mais aussi une information spatialisée sous forme de cartes et sous des formes très diversifiées selon les besoins. C'est particulièrement vrai pour les actions sur les infrastructures de voiries ou de transport en commun. L'accès aux données intermédiaires de la chaîne de modèles, en particulier sous une forme spatialisée, peut être particulièrement intéressant pour mieux comprendre et faire comprendre les mécanismes de la chaîne de causalité depuis les déterminants de la mobilité jusqu'aux impacts environnementaux et leurs conséquences. Les progrès faits en matière de couplage des modèles autour d'une infrastructure de données spatiales et du SIG open source OrbisGIS augurent bien de la possibilité d'intégrer à terme de tels outils avec le système d'information d'une collectivité et de servir ainsi des objectifs d'aménagement et de planification urbaine.

Cependant, les résultats présentés ci-dessus questionnent bien évidemment la capacité des modèles à rendre compte de changements majeurs sur les comportements de mobilité. Ceci rejoint très précisément les interrogations des chercheurs du projet eux-mêmes [3, 5, 31, 38], mises en évidence lors du travail final de retour d'expérience [6], et tout particulièrement pour ce qui concerne le modèle de trafic. La qualité intrinsèque et la sensibilité des modèles de trafic pour produire les données permettant de calculer les émissions de polluants atmosphériques ont été largement analysées par Brutti-Mairesse et al. [16, 40] et les sources d'incertitude clairement identifiées par André et Brutti-Mairesse [41].

Après la question du choix du modèle et de ses données intrinsèques, la qualité de sa mise en œuvre repose en fait sur deux piliers :

- la richesse et la précision des données amont, notamment ce qui peut être connu à partir d'une enquête ménages déplacements sur les comportements de mobilité (et leurs leviers de changement) et les choix modaux à l'échelle du territoire analysé ;

- le calage du modèle dans une situation de référence à partir de données de comptage fiables et aussi exhaustives que possible.

À partir de données amont plus riches, on peut réellement être optimiste quant à la possibilité des modèles de prendre en compte des modes ou des pratiques aujourd'hui négligés mais appelés à se développer (comme les deux-roues ou l'intermodalité). Un des freins principaux pourrait être le coût de la collecte de ces données, comme on a pu le voir avec le report sine die de l'enquête déplacement prévue initialement à Nantes en 2010. La question du coût associé à la mise en œuvre d'une chaîne de modèles telle que celle expérimentée dans le cadre du projet est d'ailleurs un obstacle majeur à son utilisation. Hors l'enquête déplacements, c'est le coût associé à la collecte, à l'achat éventuel et au prétraitement de données en grandes quantités qui pose problème (compte tenu de la nature spatiale d'une grande partie de ces informations). Mais la mise en œuvre elle-même des modèles, l'expertise requise pour les utiliser (notamment la part d'empirisme indispensable pour le choix des simplifications acceptables, des valeurs des paramètres et des relations de dépendances) et pour analyser les résultats renforcent cet obstacle.

Sur un autre plan, la phase de calage d'un modèle assure que ce modèle reproduit bien une situation de référence et elle garantit que, dans certaines limites, il reproduira correctement la sensibilité de certains scénarios. L'incertitude réside dans l'ampleur de ces limites. Un scénario qui correspondrait à une évolution très significative des déterminants de la mobilité ou des mécanismes de choix, induirait par rétroaction des mécanismes sensiblement différents de ceux modélisés et représentés par le calage : des scénarios extrêmes ou à long terme seront donc mal pris en compte, en tout cas avec une marge d'incertitude importante (quoique difficile à cerner). Il ne s'agit pas là d'une hypothèse d'école ! En effet le PDU nantais voté en 2011 met en perspective à l'horizon 2030 une mobilité accrue de $20 \%$, essentiellement à cause de la croissance de la population, avec des émissions (GES) réduites de $18 \%$ par rapport à 2008 du seul fait des actions sur les comportements (-31\% par habitant) [42]. 
Cette limite n'enlève rien à la capacité effective des modèles déjà évoquée plus haut. Mais elle rejoint la question plus générale de la confiance que peuvent leur accorder les acteurs de terrain en situation opérationnelle de décision, élus ou techniciens. Deux attitudes extrêmes peuvent être rencontrées : d'un côté la croyance absolue dans les résultats, parfois instrumentalisés lorsqu'ils confortent une volonté politique ; de l'autre le refus complet, du fait de l'incompréhension d'un modèle «boîte noire » trop mystérieux, de résultats contre-intuitifs ou contraires au projet politique. Entre les deux, il y a parfois peu de place pour une voie médiane, d'un usage raisonné, dans une logique d'apprentissage et d'appropriation de tous les acteurs, avec un accompagnement expert.

Ces deux obstacles du coût et de l'appropriation des outils incitent à travailler à moyen ou long terme au sein d'un territoire, dans une logique d'intégration des outils de simulation et des données spatiales, et d'évaluation et d'accompagnement in itinere des politiques de mobilité et, sans doute, des autres politiques urbaines en interaction avec la mobilité et les transports. C'est à la fois une condition de mise à jour progressive des données et de maintenance des outils et une condition d'une utilisation efficace par des acteurs sensibilisés et formés. Une approche ponctuelle (une évaluation une fois, et on verra peut-être dans dix ans) risque d'être contre-productive et de cumuler tous les obstacles.

\section{Remerciements}

Les auteurs remercient tous les chercheurs du programme Eval-PDU qui les ont autorisés à reproduire leurs résultats, tout particulièrement François Ducroz, Gwendall Petit et Nicolas Fortin.

Les équipes participantes citées notamment dans les schémas étaient : IRSTV, Institut de recherche en sciences et techniques de la ville, FR CNRS 2488 ; GEPEA, Génie des procédés environnement agroalimentaire, UMR CNRS 6144, Université et École des Mines de Nantes ; CEREMA (ex - Centre d'études techniques de l'équipement de l'Ouest), DVT/INT ; AirPL, Air Pays de la Loire ; IM, Division infrastructure et mobilité, équipe Acoustique, LTE, Laboratoire transports environnement, et UMRESTTE, Unité mixte de recherche épidémiologique et de surveillance transport travail environnement, Ifsttar, Institut français des sciences et techniques des transports aménagements et réseaux ; IUP, Institut d'urbanisme de Paris, Université Paris XII Créteil Marne la Vallée ; DCS, Droit et changement social, et LEMNA, Laboratoire d'économie et de management, Université de Nantes.
Cette étude a bénéficié d'un financement de Nantes Métropole et de l'Agence nationale de la recherche, programme Ville Durable (ANR-08-VILL-0005).

\section{Références}

1. Mestayer P, Abidi A, André M et al. (2012) Environmental impact assessment of urban mobility plan: a methodology including socio-economic consequences, in Urban Environment: Proc. 10th Urban Environment Symposium, S. Rauch and G.M. Morrison (eds.), Alliance for Global Sustainability Book series 19, Springer Science+Business Media B.V. (doi 10.1007/978-94-007-25409_2), pp 15-26.

2. Fouillé L, Broc JS, Bourges B et al. (2012) Eval-PDU: urban traffic and its environmental impacts modelling to assess Urban Mobility Master Plan. Conception of a methodology based on the Nantes case, In Hégron G, La modélisation des flux au service de l'aménagement urbain - Flow modeling for urban development, Ifsttar, France, pp 202-212.

3. Mestayer P (2012) Eval-PDU, Évaluation des impacts environnementaux d'un PDU et de leurs conséquences socioéconomiques: développements méthodologiques et tests sur le PDU de Nantes Métropole, Rapport scientifique final $\mathrm{du}$ Programme ANR Villes Durables ANR08-VILL-0005, www.irstv.fr/fr/recherche/contrats-de-recherche/acheves, $45 \mathrm{p}$.

4. Broc JS, Mestayer P, Bourges B et al. (2012) Évaluation environnementale des Plans de Déplacements Urbains - Séminaire organisé par l'IRSTV à Nantes Métropole. Poll Atmos 216: 333-337.

5. Abidi A, Fialaire J (2011) (dir.) Quelle gouvernance au service de la mobilité durable? L'Harmattan, $450 \mathrm{p}$.

6. Fialaire J (2011) Vers une autorité organisatrice de la mobilité durable ? In Abidi A, Fialaire J (dir), Quelle gouvernance au service de la mobilité durable? L'Harmattan, Paris, pp 189-202.

7. Fouillé L, Broc JS, Bourges B (2012) Retour d'expérience méthodologique et propositions pour une évaluation ambitieuse des impacts environnementaux des PDU. Livrable Eval-PDU L1.4, www.irstv.fr/fr/recherche/contrats-de-recherche/acheves, $80 \mathrm{p}$.

8. Moussiopoulos N (2003) Studying Atmospheric Pollution in Urban Areas, Overview of Subproject SATURN, Towards Cleaner Air for Europe - Science, Tools and Applications, P. M. Midgley $\&$ M. Reuthers edits., Margraf Publ, Weikersham, Germany, pp 227-249.

9. Borrego C, Tchepel O, Costa AM, Amorim JH, Miranda AI (2003) Emission and dispersion modelling of Lisbon air quality at local scale. Atmos Environ 37: 5197-5205.

10. Hatzopoulou M, Miller EJ, Santos B (2007). Integrating vehicle emission modelling with activity-based travel demand modelling: a case study of the Greater Toronto Area (GTA). Transp Res Rec 2011, 29-39.

11. Beckx C, Int Panis L, Van De Vel K, Arentze TA, Lefebvre W, Janssens D, Wets G (2009) The contribution of activity-based transport models to air quality modelling: A validation of the ALBATROSS-AURORA model chain. Sci Total Environ 407: 3814-3822.

12. Hatzopoulou M, Hao JY, Miller EJ (2011). Simulating the impacts of household travel on greenhouse gas emissions, urban air quality, and population exposure. Transportation 38 : 871-887.

13. Lautso K, Toivanen S (1999) SPARTACUS system for analyzing urban sustainability. Transp Res Rec 1670: 35-46. 
14. Broc JS, Bourges B (2010) Rapport méthodologique sur l'élaboration des scénarios de référence et des scénarios d'actions PDU pour l'évaluation des impacts environnementaux, Livrable Eval-PDU L1.1, www.irstv.fr/fr/recherche/contrats-derecherche/acheves, $94 \mathrm{p}$.

15. Fouillé L, Broc JS, Bourges B, Bougnol J, Mestayer P (2012) La place des modèles de trafic dans les récentes modélisations des impacts environnementaux des transports. Importance de 1'explicitation des mpthodes et hypothqses, Rech Transp Secur 28: 190-200 (doi 10.1007/s13547-012-0037-5).

16. Brutti-Mairesse E. (2013) Réflexion sur les méthodes d'évaluation environnementale des mesures de gestion des déplacements et du trafic : analyse critique et proposition d'un nouveau cadre conceptuel. Thèse de doctorat de l'ENTPE, réf. 2013-ENTP-0002. Vaulx-en-Velin, France, 378 pp.

17. Broc JS, Bourges B (2012) Description des scénarios d'actions $P D U$ pour l'évaluation des impacts environnementaux du PDU de Nantes Métropole 2000-2010, Livrable Eval-PDU L1.2, www.irstv.fr/fr/recherche/contrats-de-recherche/acheves, $89 \mathrm{p}$.

18. AURAN (2000) Agglomération nantaise, plan de déplacements urbains $2000-2010$, concilier ville mobile et ville durable. Document final approuvé par le Conseil de District du 27 octobre 2000. District - AURAN, 13 octobre 2000, 118 pp

19. Petit G (2012) How to organize the data flow of the City: a case study with the Spatial Data Infrastructure CartoPOLIS. In Hégron G, Actes, Colloque La modélisation des flux au service de l'aménagement urbain, 13-14 juin 2012, Lille, France (Poster), GIS Modélisation Urbaine. Éditions Scientifiques et Techniques IFSTTAR, Bron, France. pp 270-275 (www.cartopolis. org/).

20. Bocher E, Petit G (2012) OrbisGIS: Geographical Information System designed by and for research, in Innovative Software Development in GIS, Bucher B, Le Ber F (edits), John Wiley \& Sons, Inc., Hoboken, NJ, USA. pp 55-66 (doi: 10.1002/9781118561928.ch2).

21. Bocher E, Petit G (2012) OrbisGIS : Un Système d'Information Géographique construit par et pour la recherche. in Développement logiciels en géomatique: innovations et mutualisation, Bucher B, Le Ber $\mathrm{F}$ (Edits), Hermès-Lavoisier, Paris, pp $37-76$.

22. Circul'air version 3, ASPA 12122102-ID, http://www.atmoalsace.net

23. Pastramas N, Samaras C, Mellios G, Ntziachristos L (2014) Update of the Air Emissions Inventory Guidebook-Road Transport 2014 Update, EMISIA SA Report No: 14.RE.011.V1 (www.emisia.com/copert/).

24. Fouillé L, Rebours A, Schmidt T (2013) Modéliser les émissions et les concentrations de polluants atmosphériques pour évaluer les politiques de transport. Poll Atmos 217, 10 p. (http://lodel.irevues.inist.fr/pollution-atmospherique /index.php?id=997).

25. ATMO- France (2010) Évaluation de l'exposition des populations à la pollution atmosphprique, Guide pour une Modélisation avec une Résolution Spatiale Fine des Concentrations en Milieu Urbain, version 3 (http://www.atmo-france.org).

26. CERC (2010) ADMS 4 - Atmospheric Dispersion Modelling System-User Guide Version 4.2, Cambridge Environmental Research Consultants Ltd., Cambridge, UK.
27. Carruthers DJ, Edmunds HA, Lester AE, McHugh CA, Singles RJ (2000) Use and validation of ADMS-Urban in contrasting urban and industrial locations. Int J Environ Poll 14: 364-374.

28. BASEMIS (2013) Résultat de l'inventaire 2010. Air Pays de la Loire, EEtu13061, 51 p.

29. Sétra (2009) Guide méthodologique. Prévision du bruit routier. Volume 1 - Calcul des émissions sonores dues au trafic routier. Volume 2 - Méthode de calcul de propagation du bruit incluant les effets météorologiques (NMPB2008). English version: http://www.setra.equipement.gouv.fr/IMG/pdf/US_09572A_Road_noise_predictionDTRF.pdf

30. Picaut J, Fortin N, Dutilleux G (2011) A simplified approach for making sound maps within a GIS software, in INTER-NOISE and NOISE-CON Congress and Conference Proc. 2011(8): 147-152.

31. Picaut J, Fortin N (2012) Approche simplifiée de la réalisation de cartographies sonores de grandes agglomérations à l'aide d'un logiciel SIG, in RéférenceS | septembre 2012 | Modélisation urbaine: de la représentation au projet. Commissariat Général au Développement Durable, Paris, pp 232-235.

32. Joumard R, Gudmundsson H, Folkeson L (2011) Framework for assessing indicators of environmental impacts in the transport sector. Transp Res Rec 2242: 55-63 (doi 10.3141/2242-07).

33. Lépicier V, Chiron M, Joumard R (2012) Developing an indicator for the chronic health impact of traffic-related pollutant emissions. Environ Impact Assess Rev 38: 35-43 (doi 10.1016/j.eiar.2012.05.00).

34. Brecard D, Bulteau J (2011) Les enjeux économiques et environnementaux de la mobilité durable. In Abidi A, Fialaire $\mathrm{J}$ (dir), Quelle gouvernance au service de la mobilité durable? L'Harmattan, Paris, pp 45-62.

35. Fouillé L (2011) Indicateurs généraux de trafic 2 : Méthode simplifiée d'estimation des émissions et consommations énergétiques liées au trafic. Livrable Eval-PDU L1.4 Annexe B. www.irstv.fr/fr/recherche/contrats-de-recherche/acheves, $13 \mathrm{p}$.

36. AURAN (2009) Comment se déplace-t-on aujourd'hui dans l'agglomération nantaise et son bassin de vie? Tendances 2008. Rapport Nantes Métropole-AURAN, $40 \mathrm{p}$.

37. Brécard D, Fritsch B, Le Boennec R (2012) Plans de déplacements urbains et capitalisation immobilière: le cas des appartements de l'agglomération nantaise, Colloque de l'Association de science régionale de langue française, 9-11 juillet 2012, Belfort, p. 16.

38. Brécard D, Le Boennec R (2014) Urban transport policies and housing values: evidence from apartments in Nantes, Transp Res Part $D$ (soumis).

39. Fialaire J, Fouillé L, Karsenty S (2012) Évaluation des incidences environnementales des changements de comportement induits par les actions des PDU. Livrable Eval-PDU L6.2, www.irstv.fr/fr/recherche/contrats-de-recherche/acheves, $68 \mathrm{p}$.

40. Brutti-Mairesse E, Teillac S, André M, Leclercq L (2012) Estimation of pollutant emissions from road traffic at a city scale, and its sensitivity as regards the calibration of the static traffic assignment models, Social and Behavioral Sciences 48 : 2091-2100.

41. André M, Brutti-Mairesse E (2015) Évaluation de l'impact d'un PDU: problématique de l'émission de polluants atmosphériques, RTS 32 : 121-133.

42. AURAN (2011) Plan de déplacements urbains 2010-2015 perspectives 2030, AURAN-Nantes Métropole, juillet 2011, $134 \mathrm{p}$. 